\title{
REVIEWS
}

\section{Study on the Effect of Magnetic Fields on Polymeric Materials and Its Application}

\author{
Tsunehisa KIMURA $^{\dagger}$ \\ Department of Applied Chemistry, Tokyo Metropolitan University \\ 1-1 Minami-ohsawa, Hachioji, Tokyo 192-0397, Japan
}

(Received July 25, 2003; Accepted July 31, 2003)

\begin{abstract}
This paper reviews our recent work on the use of magnetic fields to the polymer processing. Polymers considered in this paper are not peculiar ones synthesized specially for the purpose of magnetic processing, but they are very usual ones including poly(ethylene terephthalate), polypropylene, etc. In this paper, two main magnetic effects on polymers are considered, i.e., alignment and levitation. In the first part, the magnetic torque causing the alignment is described. Then, examples of alignment in polymeric systems are presented, starting from simple cases such as magnetic alignment of fibers in a suspension to more complicated cases such as magnetic alignment of crystalline polymers in which mesophase formations and memory effects are involved. In the second part, the magnetic force acting on diamagnetic materials and its application to separation and processing of polymeric materials is described.
\end{abstract}

KEY WORDS Diamagnetism / Magnetic Alignment / Magnetic Levitation / Phase Transition /

Crystalline Polymers / Polymer Processing / Fiber /

Magnetic effects on diamagnetic materials have been known since the age of Faraday, but it is very recent that the attention has been paid to the use of these effects to the processing of diamagnetic materials including inorganic, organic, and polymeric materials. This trend is partially due to the development of superconducting technology ${ }^{1}$ that enables us to use high magnetic fields (10 T or more) in the study of materials science at individual laboratory level. Cryogen free (liquid-helium free) superconducting magnets, mostly manufactured by Japanese companies, are now on the market, with various types including a large bore type $(45 \mathrm{~cm}$ at 3.5 $\mathrm{T})$, a rotate bore type, a split type, a low fringe field type, a high field type (15 T, $52 \mathrm{~mm}$ in diameter), etc. These magnets are used in academia as well as industries for the processing purposes.

High magnetic fields provided by these superconducting magnets have made it possible to visualize the magnetic effects on "non-magnetic" materials such as diamagnetic materials. Because the diamagnetism is very small compared to the ferromagnetism, we hardly experience in daily life the effects of magnetic field on plastics, water, and living bodies, etc. Some means must be devised to visualize these effects. A straightforward way is to use high magnetic fields. If we use a superconducting magnet of $10 \mathrm{~T}$ instead of an electromagnet generating $1 \mathrm{~T}$, a small change of $1 \mathrm{~mm}$ is magnified to $10 \mathrm{~cm}$ and a phenomenon that takes a day to occur is completed in $15 \mathrm{~min}$, because the magnetic effect is proportional to the square of the magnetic flux density. Diamagnetic levitation ${ }^{2-4}$ and Moses effect ${ }^{5}$ (water surface splits in a high magnetic field) are good examples among many others. ${ }^{6-9}$

The magnetic effect on chemical reactions ${ }^{10-12}$ is one of the major fields in magnetic researches, but in this paper we are concerned with the magnetic force and the magnetic torque acting on diamagnetic materials. The origin of the diamagnetism is the induced magnetization caused by the induced motion of electrons under the applied magnetic field. ${ }^{13,14}$ The magnitude of the magnetization $M$ induced on a material is proportional to the applied field strength $H$, i.e., $M=\chi H$, where $\chi$ is the diamagnetic susceptibility. The magnitude of $\chi$ is typically the order of $-10^{-6}$ to $-10^{-5}$. Incidentally, this induced magnetization is also the source causing the chemical shift in the nuclear magnetic resonance (NMR) measurement. The interaction of $M$ with the applied field causes a repulsive force, repelling the particle away to the direction of a decreasing field strength. If this force balances with the gravitational force, then the particle levitates in the air. ${ }^{2-4}$

In the case that a material has an anisotropy in diamagnetism, a magnetic torque acts on it, resulting in rotation. The origin of the magnetic anisotropy is traced back to chemical bonds. For example, diamagnetic susceptibilities of the $\mathrm{C}-\mathrm{C}$ bond are smaller in the direction of the bond (//) than that normal to the bond $(\perp)$ $\left(\chi_{\|}<\chi_{\perp}<0\right)$, that is, the anisotropic diamagnetic susceptibility defined by $\chi_{a}=\chi_{\|}-\chi_{\perp}$ is negative. ${ }^{9}$ The $\mathrm{C}-\mathrm{C}$ bond therefore tends to align in the direction per-

†To whom correspondence should be addressed (E-mail: Kimura-tsunehisa@c.metro-u.ac.jp). 
pendicular to the applied field. An aromatic ring possesses a large diamagnetic anisotropy due to the ring current induced on the ring. As a result, aromatic rings tend to align with its ring plane parallel to the applied field.

Then, can a single benzene molecule in a liquid state align in the magnetic field? The answer is no. The magnetic energy acquired by a particle is proportional to the volume of the particle. The energy acquired by a single benzene molecule is too small compared to the thermal energy $k_{\mathrm{B}} T$ to make the Boltzmann factor large enough for an actually observable alignment. If a particle has a size large enough to overcome the thermal disturbance, it aligns under a magnetic field. A number of examples have been reported: ${ }^{9}$ organic crystals, ${ }^{15,16}$ protein crystals, ${ }^{17-19}$ carbon fibers, ${ }^{20,21}$ carbon nanotube, ${ }^{22-24}$ cellulose fibers, ${ }^{25-27}$ poly(ethylene) crystallites, ${ }^{28}$ fibrin, ${ }^{29}$ cells, ${ }^{30,31}$ and cell wall, ${ }^{32}$ etc. undergo magnetic alignment. Inorganic crystals also align under magnetic fields. ${ }^{33-37}$ Magnetic alignment of these materials is a natural result of their anisotropy in diamagnetism and their size that is large enough to exceed thermal energy. In general, crystals possess diamagnetic anisotropy ${ }^{14}$ as tabulated in literature. ${ }^{38}$

Unlike the above examples where the parameters such as the anisotropic susceptibility and the size and the shape of the aligning particle are fixed, there is another class where these parameters are not fixed but change in time, which complicates the alignment behavior. A typical example is found in the magnetic alignment of crystalline polymers during the crystallization process, where the anisotropic structures responsible for the magnetic alignment may change in size and shape in time. Discussion has been made on structure formation during polymer crystallization. ${ }^{39-49}$

The anisotropic structures are also formed transiently in many aspects of polymer phase transitions including crystallization from solutions, crystal-crystal transition, aggregation, solidification, microphase separation, electropolymerization, gellation, micell formation, and isotropic-anisotropic transition of liquid crystals, etc. These structures have a potential to undergo magnetic alignment and some of them actually undergo magnetic alignment. ${ }^{50-56}$ However, the mechanism of these transitions itself is insufficiently clarified in most cases at present time so that it is difficult to reach a unified view of magnetic alignment occurring during transitions.

Diamagnetic levitation ${ }^{2}$ is another interesting phenomenon that can be used to the polymer processing. If a diamagnetic particle is put in a field gradient, a force acts on it, repelling the particle toward a decreasing di- rection of the field strength. If this force balances with the gravitational force, the particle levitates in the air. Because the positions of the particles levitated under a field gradient are different depending on the magnetic susceptibility and the density of the particle, the levitation could be used for the separation purpose.$^{57,58}$ Of course, the use of ferromagnetism for the separation purposes is already well known. Using levitation, we can process polymeric materials under the pseudo-zero gravity environment. In the field of protein crystallization, this environment is utilized ${ }^{18,19}$ to obtain protein crystals with high qualities required for the structure determination by X-ray. A remarkable feature of the diamagnetic levitation is its trapping effect. If a field profile designed at micrometer or nanometer level is provided, diamagnetic particles of sizes down to micrometer or less are patterned according to the field profile provided. Research in this field is in progress. ${ }^{59}$

\section{DIAMAGNETIC ALIGNMENT}

A magnetization $\mathbf{M}$ induced on a material by an applied external magnetic field $\mathbf{H}$ is expressed as

$$
\mathbf{M}=\chi \mathbf{H},
$$

where the constant $\chi$ is (volumetric) diamagnetic susceptibility which is negative and dimensionless. The $\chi$ is not a scalar but a tensor if the material has anisotropy. All three principal components $\chi_{1}, \chi_{2}$, and $\chi_{3}$ of the tensor have different values in general, ${ }^{14}$ but two of them are equivalent for materials having an axial symmetry such as nematic liquid crystals, polymer fibers, etc. Namely, $\chi_{/ /}$in the direction of the axis and $\chi_{\perp}$ in the directions normal to the axis are only relevant. The interaction of $\mathbf{M}$ with the applied external field gives rise to a magnetic energy:

$$
E_{\text {mag }}=-(1 / 2) V \chi_{\perp} \mu_{0}^{-1} B^{2}-(1 / 2) V \chi_{a} \mu_{0}^{-1} B^{2} \cos ^{2} \xi
$$

where $\mu_{0}$ is the magnetic permeability of vacuum, $V$ is the volume of the particle under consideration, and $\xi$ is the angle between the magnetic flux density $\mathbf{B}$ and the particle axis $\mathbf{n}$. The dimensions of each physical constant are $\mu_{0}[\mathrm{~Wb} /(\mathrm{Am})], B\left[\mathrm{~T}=\mathrm{Wb} \mathrm{m}^{-2}\right], V\left[\mathrm{~m}^{3}\right]$, and $\chi_{a}=\chi_{\|}-\chi_{\perp}$ is dimensionless. Then, $E_{\text {mag }}$ is expressed by [J], accordingly. Here, approximation of $B=\mu_{0} H$ is used. The second term of the above equation is orientation-dependent, being the source of magnetic alignment. If $\chi_{a}$ is positive, the alignment of the axis parallel to the field direction is preferred to the perpendicular alignment. A macroscopic alignment could occur when the second term exceeds the thermal energy 


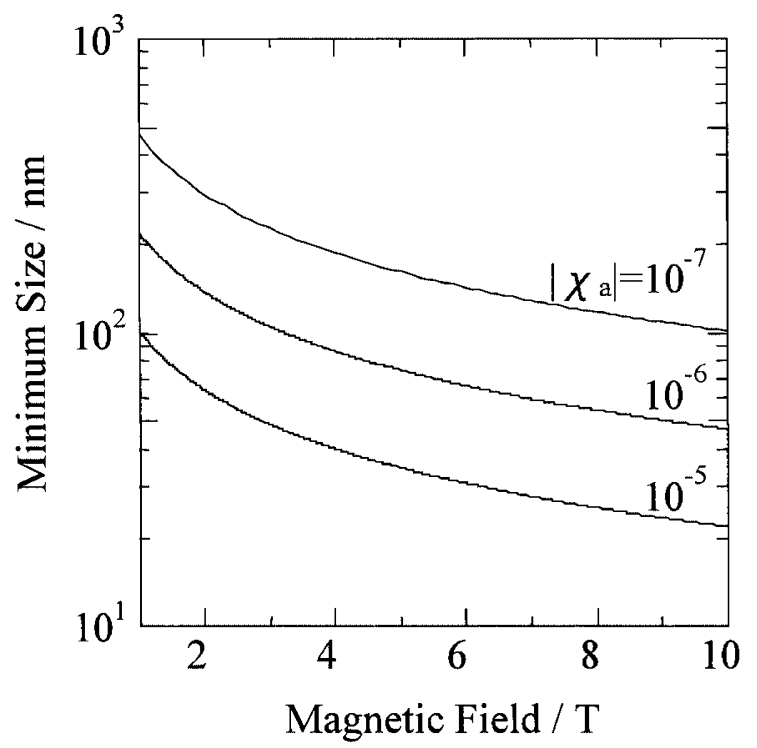

Figure 1. Minimum particle size necessary to exceed the thermal energy. Estimation by eq 3 with $T=300 \mathrm{~K}$ for various values of $\left|\chi_{a}\right| \mathrm{s}$.

$k_{\mathrm{B}} T$. This condition is expressed as

$$
V>2 k_{\mathrm{B}} T \mu_{0} /\left|\chi_{a}\right| B^{2} .
$$

From this equation, we estimate a minimum critical volume necessary for the alignment as a function of the applied magnetic flux density $B$ and the material constant $\chi_{a}$. The result is displayed in Figure 1.

The torque $\mathbf{N}$ acting on the particle is expressed as

$$
\mathbf{N}=V \chi_{a} \mu_{0}^{-1} B^{2} \sin \xi \cos \xi \boldsymbol{\omega},
$$

where is $\boldsymbol{\omega}$ a unit vector normal to $\mathbf{n}$ and $\mathbf{B}$, and $\xi$ is the angle between $\mathbf{n}$ and $\mathbf{B}$. If the particle is immersed in a viscous liquid of viscosity $\eta$, the particle rotation is governed by the balance of the magnetic torque and the hydrodynamic torque as follows:

$$
L(d \xi / d t)=-V \chi_{a} \mu_{0}^{-1} B^{2} \sin \xi \cos \xi,
$$

with the left hand side representing the hydrodynamic torque. The solution for this equation is given by

$$
\tan \xi=\tan \xi_{0} \exp (-t / \tau),
$$

with the alignment rate $\tau^{-1}$ being defined as

$$
\tau^{-1}=(V / L) \mu_{0}^{-1} \chi_{a} B^{2}
$$

In the case of a sphere of radius $a$, we have $L=8 \pi \eta a^{3}$ and $V=(4 / 3) \pi a^{3}$, to obtain

$$
\tau^{-1}=\mu_{0}^{-1} \chi_{a} B^{2} / 6 \eta .
$$

It should be noted that the above picture is valid only for a particle whose size is large enough to overcome the thermal disturbance. It should be also noted in eq 8 that the alignment rate does not depend on the volume of

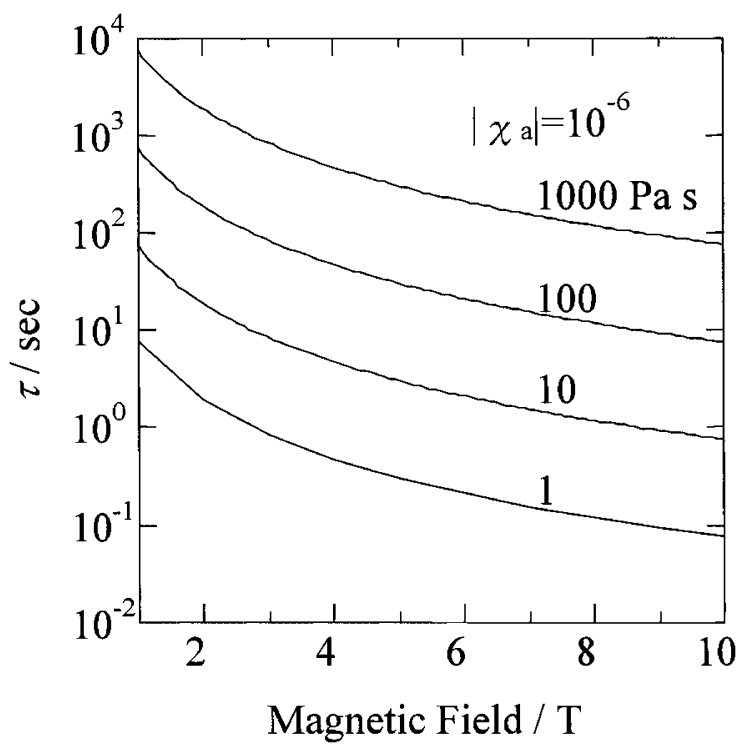

Figure 2. Time required for the magnetic alignment of a particle suspended in a liquid medium of viscosities indicated in the figure. Estimation by eq 8 with anisotropic diamagnetic susceptibility of $\left|\chi_{a}\right|=10^{-6}$.

the particle. In Figure 2, $\tau$ is plotted as a function of the magnetic flux density for various values of viscosity.

To summarize, the conditions necessary for the magnetic alignment to occur are (i) the particle should have anisotropic diamagnetic susceptibility, (ii) the size of the particle is large enough to overcome the thermal energy, and (iii) the viscosity of the surrounding medium should be low.

\section{FIBER AND CRYSTAL ALIGNMENT IN LIQUID SUSPENSIONS}

\section{Fiber Alignment}

Magnetic alignment of fibers ${ }^{20,21,25,60}$ suspended in a liquid medium is a good example to demonstrate the validity of the theoretical considerations described above. Unlike a simple case of a sphere particle described by eq 8 , the magnetic alignment of a fiber is complicated because the hydrodynamic toque appearing in eq 5 is not as simple as that for the case of a sphere. If we assume that a fiber has a prolate shape, the hydrodynamic term $L$ in eq 5 is given by

$$
L=8 \pi \eta a^{3} D / F(D),
$$

with a front factor $F(D)$ being a function of the aspect ratio of the particle. ${ }^{60-63}$ Then, the alignment rate is given by

$$
\tau^{-1}=F(D) \mu_{0}^{-1} \chi_{a} B^{2} / 6 \eta .
$$

A polyethylene (PE) fiber and a carbon fiber were used for experiment. ${ }^{60}$ A superdrawn polyethylene fiber with diameter of $30 \mu \mathrm{m}$ was cut into 1, 2, 3, and 


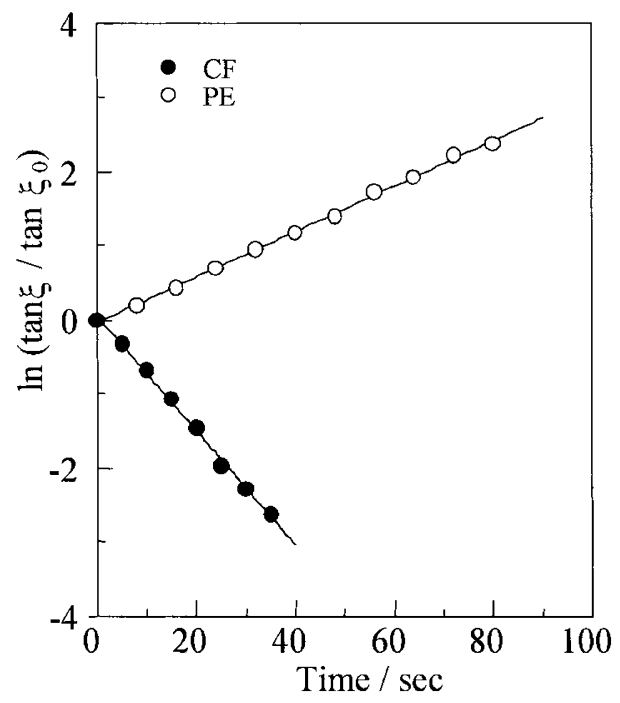

Figure 3. Measurement of fiber rotation. The angle $\xi$ of the fiber axis with respect to the field direction is plotted against the time. The origin of the time corresponds to the start of the application of the field. CF and PE indicate carbon fiber and polyethylene fiber, respectively. The slope is positive for PE because it aligns perpendicular to the applied field.

$4 \mathrm{~mm}$ lengths and suspended in a water/ethanol mixture having the same density as the fiber. The viscosity of the mixture was $2.75 \times 10^{-3} \mathrm{~Pa}$ s. A carbon fiber with diameter of $10 \mu \mathrm{m}$ was cut into $1,2,3$, and $4 \mathrm{~mm}$ lengths and suspended in a carbon tetrachloride/1,2 dibromoethane mixture ( $1 / 5$ by volume) having the same density as the fiber. The viscosity of the mixture was $1.06 \times 10^{-3} \mathrm{~Pa}$ s. A glass cell containing the suspension was put in the center of an electromagnet generating horizontal magnetic field up to $1.2 \mathrm{~T}$. The rotation of a fiber was monitored by a CCD camera and recorded on a computer. The angle $\xi$ of the fiber axis with respect to the field direction was determined as a function of time.

Figure 3 shows the logarithmic plot of $\tan \xi$ against the time. From the slope the value of $-\tau^{-1}$ is determined. The PE fiber exhibits a negative slope because the $\chi_{a}$ of the PE fiber is negative (the fiber aligns perpendicular to the field). The experimental values of $B$, $\eta, D$, and $\tau$ being known, $F(D)$ is compared with the experiment using eq 10 with an appropriate shift by a factor of $\chi_{a}$. Figure 4 shows the result. The fitting is good and the shift factors for each fiber lead to the values $\chi_{a}=-7.7 \times 10^{-7}$ and $4.0 \times 10^{-6}$ for PE and CF, respectively.

The values of $\chi_{a}$ are also determined by other methods. Those are (i) the direct measurement of the magnetic torque by means of torque meter and (ii) the separate measurements of $\chi_{/ /}$and $\chi_{\perp}$ by means of superconducting quantum interference device (SQUID) to obtain the difference. Unlike our method, both of these methods are not applied for a single fiber but they need a

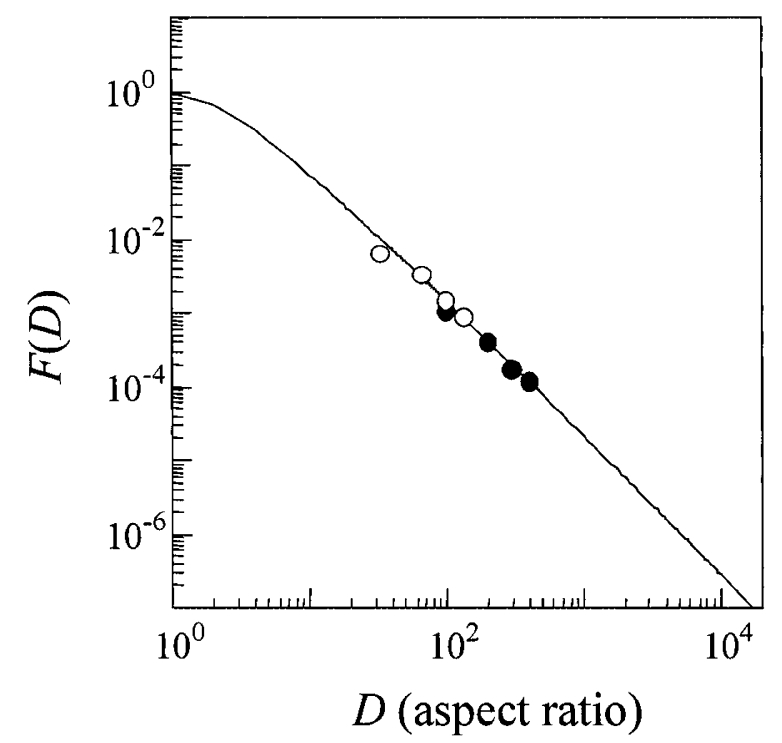

Figure 4. The value of $\tau^{-1}\left(\mu_{0}^{-1} \chi_{a} B^{2} / 6 \eta\right)^{-1}$, which corresponds to the front factor $F(D)$ in eq 10 , is plotted against the aspect ratio $D$ of the fiber. Solid line is a theoretical curve for $F(D)$ evaluated under assumption that a fiber is approximated by a prolate ellipsoid. Experimental data for carbon fiber $(\mathrm{CF})$ and polyethylene fiber (PE) are shifted by a factor $\chi_{a}$ to be fitted onto the theoretical curve, from which the value of each $\chi_{a}$ is determined.

Table I. Comparison of the anisotropic diamagnetic susceptibility determined by different methods ${ }^{64}$

\begin{tabular}{cccc}
\hline & Suspension Method & Torque Meter & SQUID \\
\hline PBO fiber & $6.6 \times 10^{-6}$ & $7.7 \times 10^{-6}$ & $7.0 \times 10^{-6}$ \\
Carbon fiber & $8.0 \times 10^{-6}$ & $7.2 \times 10^{-6}$ & $8.9 \times 10^{-6}$ \\
\hline
\end{tabular}

bundle of aligned fibers. Table I compares the values of $\chi_{a}$ of the carbon fiber and the poly ( $p$-phenylene-2,6benzobisoxazole) (PBO) fiber obtained by the different methods. The results are in good agreement. ${ }^{64,65}$

Fiber alignments are also possible for suspensions with much higher fiber concentrations. Figure 5 shows short PBO fibers magnetically aligned perpendicular to the film surface followed by solidification of the matrix liquid by polymerization. A similar experiment is also carried out for other organic fibers. ${ }^{66,67}$ The thermal conductivity of the carbon fiber is higher in the direction of the fiber axis than in the direction perpendicular to the axis. Then, if the carbon fibers are aligned perpendicular to the film surface, the thermal conductivity through the film is much improved compared to the case of random orientation.

\section{Crystal Alignment}

Crystallites of micrometer sizes suspended in a liquid align very easily even under moderate magnetic fields. In an attempt to fabricate a diffusion type polarizer, ${ }^{68}$ urea crystallites prepared by recrystallization method were magnetically aligned. ${ }^{69}$ The urea crystallites sus- 


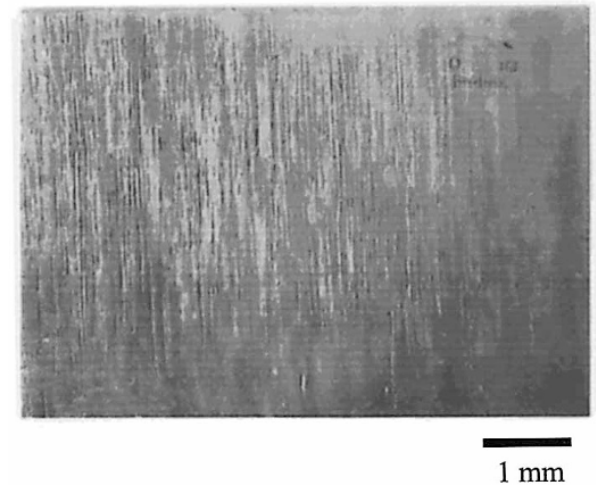

Figure 5. Magnetic alignment of short PBO fiber suspension with much higher fiber concentration. Field direction is normal to the film surface. By courtesy of M. Tobita.

pended in toluene solution of poly(ethyl methacrylate) (PEMA) was put in a magnetic field (higher than $0.5 \mathrm{~T}$ ) and allowed to evaporate toluene to obtain a PEMA/aligned urea crystal composite film. Microscope observation showed that the $c$-axis of the urea crystal aligned parallel to the field direction, indicating that this crystallographic axis corresponds to one of the principal magnetic susceptibility axes, that is, the one associated with the smallest $|\chi|$ value. The urea crystal has two refractive indices, one for the ordinary light $\left(n_{\mathrm{o}}=1.484\right)$ and the other for the extraordinary light $\left(n_{\mathrm{e}}=1.602\right)$. The direction for $n_{\mathrm{e}}$ coincides with the $c$-axis. According to the crystal symmetry, ${ }^{70}$ the principal axes of the diamagnetic susceptibility tensor and those of the refractive index tensor are in parallel relation. In addition, these axes are further related to the crystallographic axes depending on the crystal type. ${ }^{14}$ Because the difference between $n_{\mathrm{e}}$ and the refractive index of PEMA $\left(n_{\mathrm{m}}=1.4778\right)$ is large, the component corresponding to the extraordinary light in the impinging light is selectively scattered by aligned urea crystallites during the light propagates through the film, while little scattering occurs to the ordinary light, resulting in polarization in the transmitting light.

Crystallites also align in polymer melts. Because the melt viscosity is much higher than the viscosity of suspensions, higher magnetic fields are necessary to attain a quick alignment. We aligned crystallites of $N, N^{\prime}$-dicyclohexyl-2,6-naphthalenedicarboxamide $(\text { DCNDCA })^{71}$ in a melt of isotactic polypropylene (iPP). ${ }^{72}$ DCNDCA is a nucleating agent that encourages the epitaxial growth of the $\beta$-phase iPP. As will be described later, the alignment of the $\beta$-phase iPP was induced by the magnetic alignment of the DCNDCA.

A number of studies have been reported about magnetic alignment of crystals. A magnetic field up to 10 $\mathrm{T}$ was applied during the drying process of the slurry composed of an alumina powder, a dispersant, and wa-

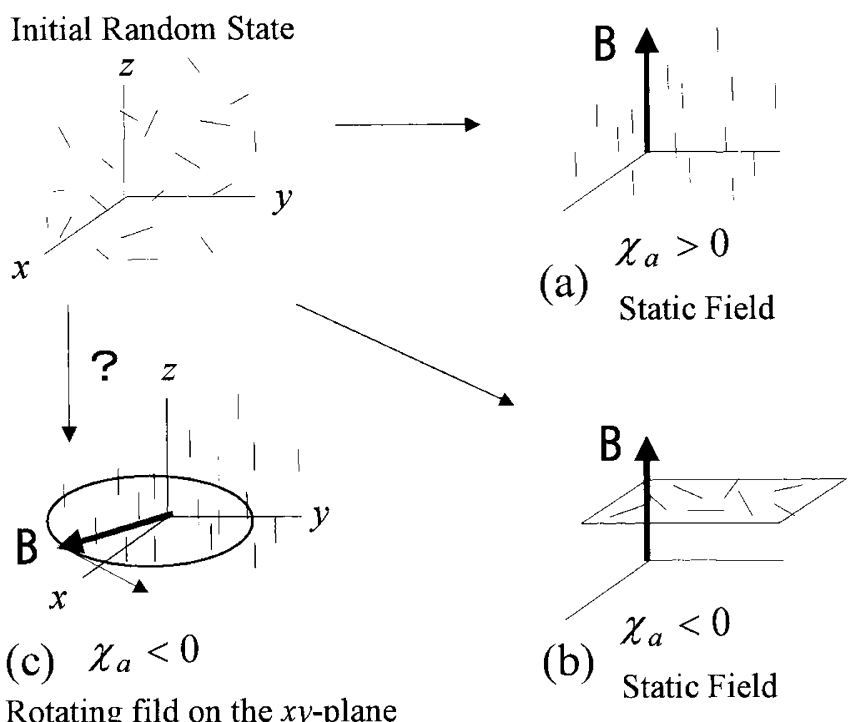

Rotating fild on the $x y$-plane

Figure 6. Alignment of an anisotropic particle with (a) $\chi_{a}>0$ and (b) $\chi_{a}<0$ under a static field and (c) $\chi_{a}<0$ under a rotating field. Short lines indicate the direction of the anisotropic axis.

ter to produce a green compact. The obtained compact exhibited alignment with the $c$-axis of the alumina grains oriented parallel to the applied field. The sintered body of the compact exhibited the grain alignment and the grain growth. ${ }^{34,73,74}$ Organic compounds ${ }^{15,16}$ and proteins ${ }^{17,18}$ are reported to align when crystallized from solutions under magnetic fields.

\section{Precision Alignment}

We have so far considered the alignment under a static magnetic field. Here we demonstrate that the use of time-dependent magnetic fields provides a powerful means to achieve the alignment difficult or impossible to realize by means of a static field alone.

Fibers with $\chi_{a} \equiv \chi_{\|}-\chi_{\perp}>0$ align parallel to the applied field, while those with $\chi_{a}<0$ align perpendicular to the field. Carbon fibers and PBO fibers for example belong to the first category, while polyethylene (PE) and cellulose fibers ${ }^{25}$ belong to the latter. If a static field is applied to carbon fibers suspended randomly in a liquid, all of their axes finally align in the direction of the applied field and hence the uniaxial alignment is attained (Figure 6(a)). On the other hand, if a static magnetic field is applied to PE fibers suspended randomly in a liquid, they align on the plane perpendicular to the field, but the directions of the individual fiber axes on this plane are random (Figure $6(b)$ ). This is in marked contrast to the case of carbon fibers where a uniaxial alignment is realized.

If a magnetic field rotating on the $x-y$ plane is applied to a suspension of fibers with $\chi_{a}<0$ (Figure 6(c)), what would happen? Because the individual fibers tend to align perpendicular to the field whether the field 


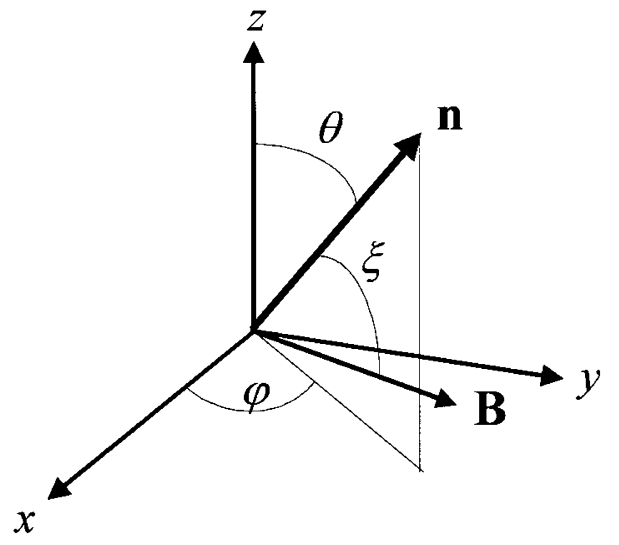

Figure 7. Field $\mathbf{B}$ rotating on the $x y$-plane is applied to a spherical particle with the anisotropic axis $\mathbf{n}$. Sufficiently long time after the start of field application, the angle $\xi$ between $\mathbf{B}$ and $\mathbf{n}$ becomes $90^{\circ}, \varphi$ follows the field rotation with $\pi / 2$ or $-\pi / 2$ phase shift, and $\theta$ depends on the initial angles and rotation speed.

is rotating or static, it is expected that the fibers align in the direction of the $z$-axis, i.e. perpendicular to the $x-y$ plane. Based on eq 5 with a simple case of a sphere, a simulation was carried out ${ }^{75}$ on a sphere of $\chi_{a}<0$ subjected to a magnetic field $\mathbf{B}$ rotating on the $x-y$ plane at the angular velocity of $\omega$. Temporal change of the direction of the anisotropic axis (represented by $\mathbf{n}$ in Figure 7) is described in terms of the time course of the angles $\theta$ and $\varphi$. Depending on the initial values of the $\theta$ and $\varphi$, the stationary value of $\theta$ is different. For example, in the case (i) that starts with $\theta=60^{\circ}$ and $\varphi=30^{\circ}$, the value of $\theta$ ends up with $90^{\circ}$. On the other hand, if we start with (ii) $\theta=60^{\circ}$ and $\varphi=80^{\circ}$, the value of $\theta$ ends up with $c a \cdot 11^{\circ}$, close to the $z$-axis. The final direction of $\mathbf{n}$ is dependent on the initial values. Also the parameters $\tau^{-1}=\mu_{0}^{-1} \chi_{a} B^{2} / 6 \eta$ and $\omega$ influences the final results. Detailed analyses will be reported elsewhere.

A much more straightforward way is to use a doubleor multiple-step method where the static field is alternately applied to the sample from two different directions perpendicular to each other. A simulation shows that this sort of time-dependent magnetic fields brings about a uniaxial alignment of PE type fibers $\left(\chi_{a}<0\right)$ irrespective of the initial distribution of the fiber orientation. In general, the use of the time-dependent magnetic field is useful when one wants to align the axes of the negative largest $\chi$, instead of the smallest one, in a uniaxial manner. These include the alignment of crystals, liquid crystals, liquid crystalline polymers, etc. The detail will be reported elsewhere.

\section{MAGNETIC ALIGNMENT OF LIQUID CRYSTALLINE POLYMERS}

\section{Domain Model for Alignment}

It is well known that liquid crystalline polymers (LCP) align under magnetic fields. ${ }^{76-87}$ The origin of the magnetic alignment is a "domain" within which LCP chains are aligned in the same direction and capable of rotating cooperatively by a magnetic torque. The size and the shape of the domain are not defined clearly because the director field defining the alignment of a liquid crystalline system is continuous except at disclinations. However, it would be allowed to the first approximation to regard a liquid crystalline system as being an assembly of randomly oriented domains that can respond to the applied field independently. Of course, this view is insufficient in higher approximations because the size and the shape of the domains would change in accordance with the change in location of disclinations during the magnetic alignment. This aspect of liquid crystalline systems is in marked contrast to the suspension systems in which the size and the shape of the suspended particles remain unchanged during the magnetic alignment.

Starting with the initial random distribution of the domain director $\mathbf{n}$, one can calculate the temporal development of the distribution function $P(\xi)$ by applying eq 6 to the individual domains. Here, $\xi$ is the angle between the director $\mathbf{n}$ and the magnetic field $\mathbf{B}$. In the two-dimensional case, $P(\xi)$ is given as ${ }^{79,86}$

$$
P(\xi)=\frac{\exp (t / \tau) \sec ^{2} \xi}{2 \pi\left(1+\exp (2 t / \tau) \tan ^{2} \xi\right)}
$$

This function is directly related to the X-ray azimuthal profile at time $t$ after the start of the application of the field, so that the alignment speed $\tau^{-1}$ is determined by fitting to the X-ray data.

The domain model does not work very well for the description of the realignment experiment. This experiment includes (i) the alignment from the initial random orientation, (ii) followed by switching of the field direction by $90^{\circ}$ (realignment). Figure 8 (a) shows the function $P(\xi)$ during the realignment process. The azimuthal peak centered at $0^{\circ}$ disappears then is rebuilt up at $\pm 90^{\circ}$. This prediction fails to explain the experimental result shown in Figure 8 (b), where two azimuthal peaks around $50^{\circ}$ appear before the peaks at $\pm 90^{\circ}$ are finally built up. The reason for the failure is that the interaction between domains is not included in the domain model. In fact, a simulation that includes the elastic interaction between domains can reproduce the behavior observed in experiment (Figure $8(\mathrm{c})) .{ }^{86}$ 

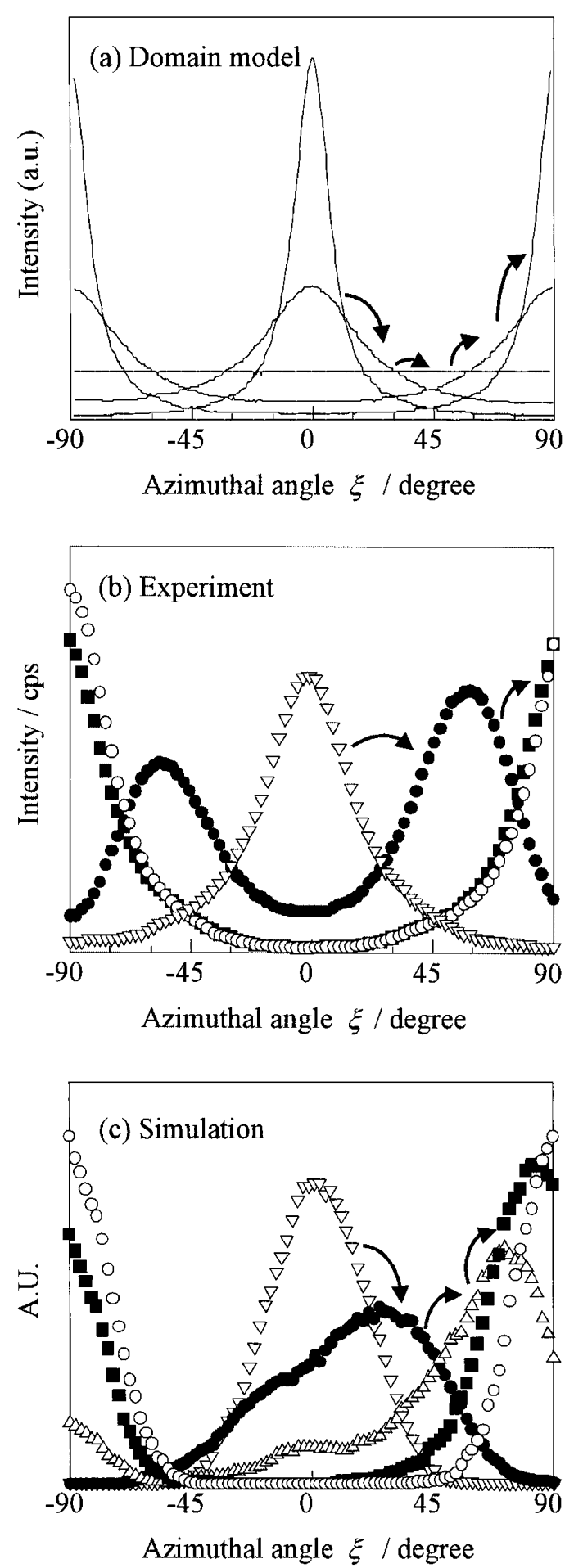

Figure 8. Change in X-Ray azimuthal profiles during the realignment process. Prediction by (a) the domain model, (b) the experimental results obtained for a liquid crystalline polymer (Rodrun LC3000), and (c) the result of simulation which includes the interaction between domains. Temporal profile changes are indicated by arrows.

\section{Mechanical Properties of Magnetically Aligned Liquid Crystalline Polymer}

High alignment of liquid crystalline polymers (LCPs) and resultant mechanical properties are attained using magnetic fields. There are several advantages of using magnetic fields for the alignment of LCPs over the use of mechanical alignment including stretching and shearing. ${ }^{81-84}$ Because magnetic fields penetrate into materials, the alignment is attained throughout the thickness. In addition, the direction and the strength of the field are controlled easily in arbitrary manners, and the alignment of LCP chains (most LCPs having aromatic rings in the main chain, they align with their chain axis parallel to the field), accordingly. The designed alignment is usually difficult by means of mechanical methods. By magnetic alignment, chain conformation is little affected because the orientation is attained by the rotation of "domains", while by mechanical alignment, chains are elongated, causing conformation changes.

Because the orientation mechanism is entirely different between magnetic and mechanical methods, it is important to clarify the intrinsic mechanical properties attained by means of the magnetic alignment. For this purpose, comparison was made of mechanical properties of films aligned by means of mechanical and magnetic methods. ${ }^{83}$ A Unitika random copolyester Rodrun LC-3000 composed of $60 \mathrm{~mol} \%$ $p$-hydroxybenzoic acid (PHB) and $40 \mathrm{~mol} \%$ ethylene terephthalate (ET) was heat-treated in a magnetic field (6 T) at temperatures between 231 and $251^{\circ} \mathrm{C}$ for 15 to $120 \mathrm{~min}$ to obtain aligned films with various orientation degrees. The orientation degree was determined using the half width of the X-Ray azimuthal peak. Mechanically elongated film samples were also prepared. These mechanically prepared film samples were thin $(30-50 \mu \mathrm{m})$ enough to avoid a skin-core structure often encountered in thick film and sheet samples. The obtained films were subjected to the tensile test.

Figures 9 (a) (c) show the elastic modulus, the tensile strength, and the elongation to break, respectively, as a function of the orientation degree. The elastic modulus for the magnetically aligned films shows a similar behavior to that for the mechanically elongated ones. On the other hand, the tensile strength and the elongation to break are different between two methods of alignment. Low values of the tensile strength for the magnetically aligned films could be attributed to the inhomogeneous phase structure observed with polarizing microscope. Many spots of $10-20 \mu \mathrm{m}$ size were observed, which were rich in ET component as revealed by microscope-FT-IR. These spots occurred due to the micro-phase separation ${ }^{88}$ during a prolonged heat treatment time necessary for the magnetic alignment.

Microscopic investigation was also carried out using X-Ray, ${ }^{13} \mathrm{C}$ solid-state NMR, and FT-IR. The spacer (ET) conformation of the magnetically aligned film was similar to that of heat-treated film without magnetic 

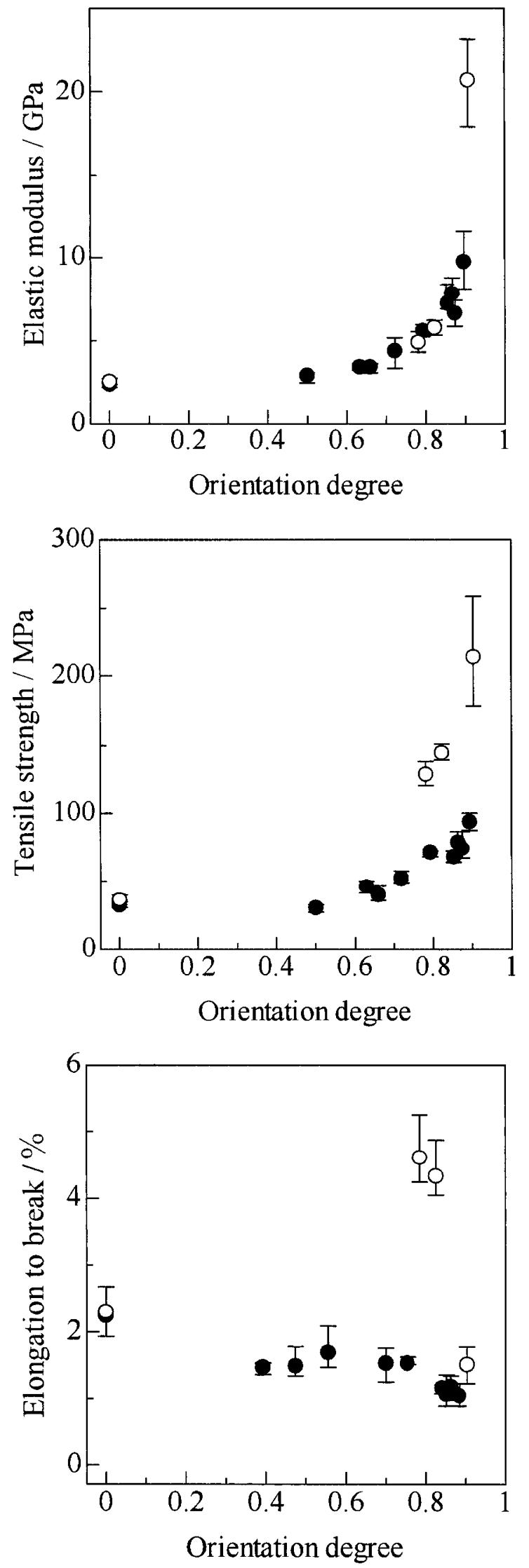

Figure 9. Mechanical properties of magnetically ( $\bullet$ ) and mechanically $(\circ)$ aligned liquid crystalline polymer (Rodrun LC3000): (a) Elastic modulus, (b) tensile strength, and (c) elongation to break. (a)

(b)
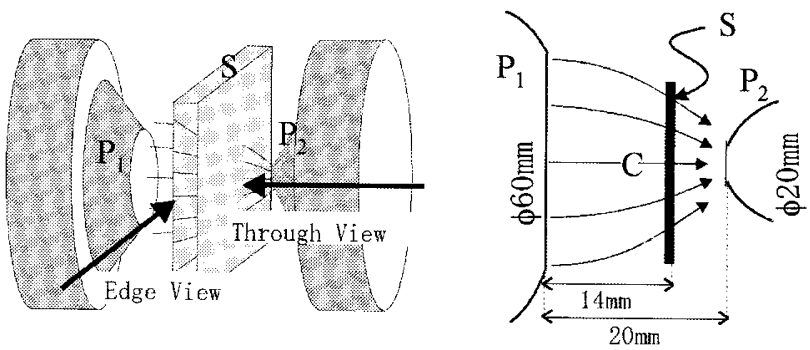

Figure 10. (a) Schematic diagram of an electromagnet with pole pieces $P_{1}$ and $P_{2}$ with different sizes. $S$ indicates the sample. (b) Actual geometry of the sample location. $\mathrm{C}$ indicates the center of the sample.

field. They were slightly rich in trans-form than the mechanically elongated film. The mesogen (PHB) conformation of the magnetically aligned film was similar to that of the as-pressed film. These observations indicate that the applied magnetic field does not alter the chain conformation, but it just rotate the "domain". Studies on mechanical and therma ${ }^{85}$ properties of another commercial LCP (Xydar) were also carried out. ${ }^{81,82}$

\section{Graded Alignment of Liquid Crystalline Polymer}

Using a magnetic field of a designed field profile, one can obtain a designed alignment profile of LCP chains in a film and a bulk. Here we demonstrate a graded alignment ${ }^{89}$ realized by an electromagnet having asymmetric pole pieces. Figure 10 (a) shows the electromagnet used for the purpose. Two pole pieces with different diameters are set to generate graded field profile schematically shown in Figure 10 (b). An LCP (Rodrun LC-3000) pellets were hot-pressed to obtain a film which was screwed between a set of heater plates and then placed between the pole pieces, and heated at $280^{\circ} \mathrm{C}$ for $20 \mathrm{~min}$. The field strength around the sample center was $c a .2 .4 \mathrm{~T}$. Then, the sample was cooled and released from the heater and subjected to the X-Ray measurement. The X-Ray measurement was carried out at the edge view (Figure 10 (a)).

Figures 11 (a), (b) show X-Ray diagrams. Figure 11 (a) shows that in the initial pressed film the chains are aligned parallel to the film surface. Upon application of the graded field, it is found in Figure 11 (b) that the chains are aligned almost perpendicular to the film at the region close to the center of the film. Above and below the center, the chains are tilted with respect to the direction normal to the film surface. The tilt angle is in good agreement with the field profile calculated by simulation. 

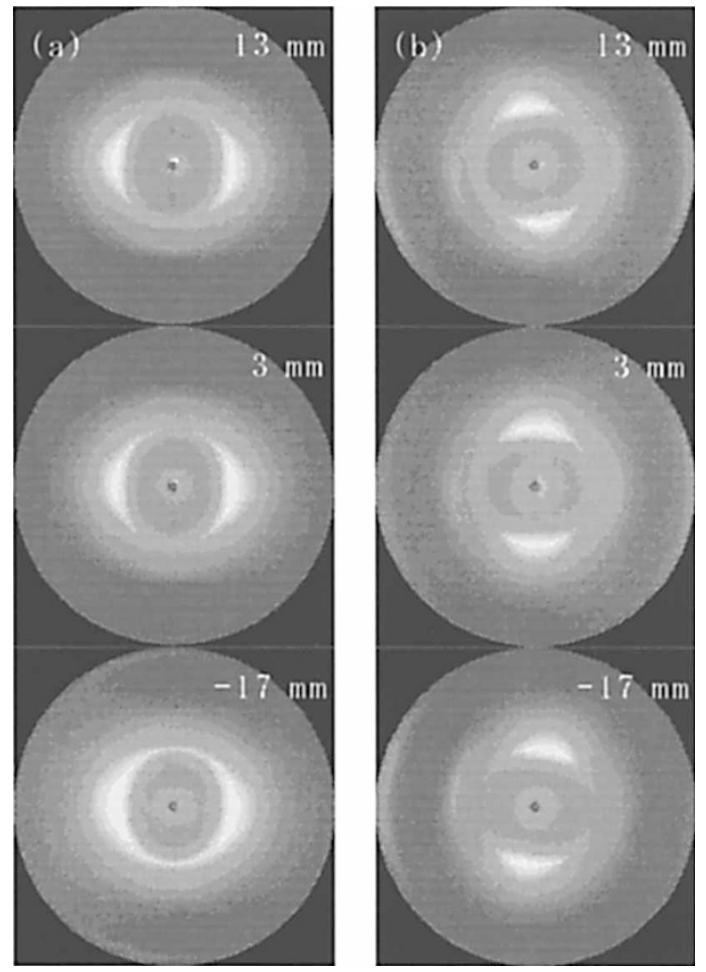

Figure 11. X-Ray diagrams (edge views) obtained at various sampling positions indicated in the figure, measured from the sample center C, taken (a) before and (b) after the heat treatment in the magnetic field (2.4 $\mathrm{T}$ at the sample center).

\section{MAGNETIC ALIGNMENT OF CRYSTALLINE POLYMERS}

\section{General View}

In contrast to the liquid crystalline polymers, crystalline polymers were considered to be unable to undergo magnetic alignment because they lack a liquid crystalline phase necessary to respond to the applied field. They may not align in their molten and solution states because these states are assumed to be isotropic. Nor may they align in their solid states (glassy and semi-crystalline states) because the viscosity is too high. However, we have found that many crystalline polymers including poly(ethylene-2,6-naphthalate) $(\mathrm{PEN}),{ }^{90,91}$ isotactic polystyrene (iPS), ${ }^{92}$ isotactic polypropylene (iPP), ${ }^{93}$ poly(ethylene terephthalate) (PET) ${ }^{94}$ paraffin, ${ }^{95,96}$ poly(ethylene oxide) $(\mathrm{PEO}){ }^{97}$ polyetherester, ${ }^{98}$ poly(carbonate) (PC),${ }^{99}$ and cellulose triacetate (CTA) ${ }^{100}$ align magnetically under appropriate conditions.

A common feature of the alignment conditions is that the alignment occurs during the transitions between the solid and liquid states such as crystallization from melt as well as melting from crystal. Because some structure of critical size with anisotropic diamagnetic susceptibility existing in a relatively low viscos- ity environment is essential for the magnetic alignment to occur, the observation of magnetic alignment strongly suggests that some ordered structure (hereafter referred to as mesophase) is involved during these transitions. In some cases, the alignment is observed in the molten state, indicating that mesophase exists even in the melt. ${ }^{101}$ At present, we do not fully identify the mesophase responsible for the magnetic alignment. It may be a crystal embryo, a microcrystallite undetectable by X-Ray, a nematic-like structure, a condis crystal, ${ }^{102,103}$ and so forth, or a mixture of these. Further studies will be necessary regarding this point. For deeper understandings of magnetic alignment, understanding of the crystallization itself is inevitable, but no unified view about the order formation during polymer crystallization seems to be reached. ${ }^{39}$

Practically, the key point for the attainment of a magnetic alignment is to encourage the mesophase formation. Though the nature of the mesophase itself in not fully understood yet as mentioned above, there are some hints. Because polymeric systems have long relaxation times, the phase transitions are not completed instantly but usually take a time. As a result, some orders existing in the crystal could remain for some while even after the temperature passes through the melting point $T_{\mathrm{m}}$. The same thing could happen when the crystal is dissolved to a solution. This is usually termed as "memory effect", and the residual ordered structures could be referred to as mesophase. The mesophase may be transient or even stable thermodynamically in a limited temperature range. If the magnetic field is applied before the memory effect disappears, the chance of magnetic alignment would be high. When polymer chains are crystallized from a melt or a solution, they have to disentangle from each other. And it will take some time for that. If the field is applied at time when the viscosity is still low and mesophase is appropriately developed, the chance to succeed in alignment would be high.

A general thermal scheme used to attain magnetic alignment during melt crystallization is shown in Figure 12. Here, the field is applied throughout the whole process. A solid sample is heated from room temperature to a maximum melting temperature $T_{\max }$ above the melting point $T_{\mathrm{m}}$. Then the sample is kept melted for a period of $t_{\mathrm{m}}$, followed by cooling down to the temperature $T_{\mathrm{c}}$ for the crystallization. The size of mesophase domain and the viscosity of the environment are two major important factors. Also important are other factors including the solid structure before the melting, the life time of mesophase, and the formation of tertiary structures such as spherulite that usually obscures the alignment. Although the detailed conditions depend 


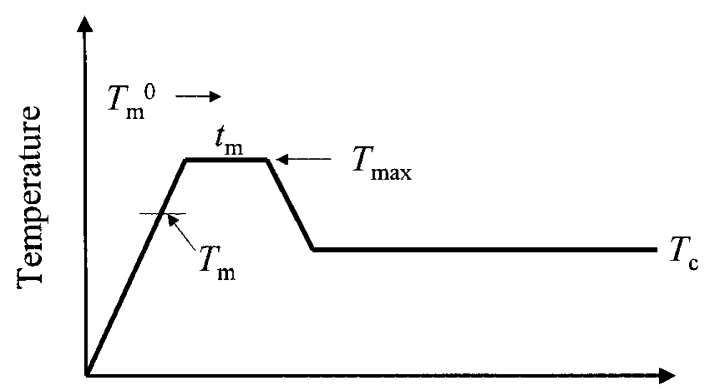

T i m e

Figure 12. General thermal scheme used to attain magnetic alignment during the crystallization from melt. Magnetic field is applied throughout the process. RT: room temperature; $T_{\mathrm{m}}$ : melting point of the polymer; $T_{\max }$ : the maximum temperature at which the sample is melted; $T_{\mathrm{c}}$ : crystallization temperature; $t_{\mathrm{m}}$ : melting time.

from polymer to polymer, important points are summarized as follows:

(1) Solid state of the initial film. The initial state could be semi-crystalline or glass. The initial state and subsequent heating process affect the solid structure reached just before the melting and hence they affect the residual structure (mesophase) in the melt.

(2) Heating process. During this process, glass films could develop crystallites due to cold crystallization. If the heating rate is too high or the cold crystallization is too slow, there is no chance to develop crystallites. Crystallites formation is important because crystallites formed would encourage the formation of mesophase in the subsequent process.

(3) Melting temperature, $T_{\max }$. This temperature should be between the melting point $T_{\mathrm{m}}$ (defined by the top of the endothermic peak at DSC measurement) and the equilibrium melting point $T_{\mathrm{m}}{ }^{0}$. If the sample is heated at high temperatures for a prolonged time, residual mesophase originating from the solid state disappears completely. As a result, the mesophase alignment at this stage and the stage of further structure formation in the subsequent crystallization process become insufficient.

(4) Crystallization temperature, $T_{\mathrm{c}}$. The mesophase alignment develops in the early stage of crystallization (induction period) where no crystallites are identified by means of wide angle $\mathrm{X}$-ray diffraction. If $T_{\mathrm{c}}$ is too low, crystallization is completed before alignment develops. If $T_{\mathrm{c}}$ is high, spherulites develop to obscure the alignment.

Additional comments should be made about some observations that are not fully interpreted at present. The first comment is about the molecular weight dependence. We have found so far that a low molecular weight iPP undergoes magnetic alignment ${ }^{93}$ but high molecular ones do not. ${ }^{104}$ Paraffin aligns ${ }^{95}$ but polyethylene, as far as we have tried, does not align. Also, low molecular weight PEO aligns from a melt, ${ }^{105}$ but high molecular one hardly aligns. PEN ${ }^{106}$ and PET with the molecular weight ranging $c a .8000$ to 20000 align. The alignment seems to become difficult if the molecular weight is increased. However, iPS $^{92}$ of the nominal molecular weight of 400000 aligns. These observations do not seem to be interpreted simply in terms of viscosity effect. There might be unknown factors.

The second comment is about a direct magnetic effect on crystallization, that is, the rate of crystallization or the perfection of crystals. Our study on iPS indicates that the crystallization is accelerated ${ }^{92}$ The lamella perfection of iPS is higher when crystallization is carried out in the magnetic field. ${ }^{39}$ The interpretation of these observations might be related to the third comment.

The third comment is that the magnetic free energy that the mesophase or the crystals have is orientationdependent so that the applied field could affect the crystallization behavior itself including the rates of nucleation and growth. In fact, the magnetic field causes the shift of the melting point ${ }^{107-109}$ depending on the mutual orientation of crystals with respect to the applied field, as will be discussed later.

\section{Magnetic Alignment of Poly(ethylene terepthalate)}

In this section, the magnetic alignment of poly(ethylene terephthalate) (PET) is presented as an example of magnetic alignment of crystalline polymers. ${ }^{94,110}$ In-situ magnetic birefringence measurement was carried out for poly(ethylene terephthalate) (PET) together with X-ray analyses of the samples obtained by interrupting the heat treatment by quenching. Pellets of PET $\left(M_{\mathrm{w}}=c a\right.$. 10000) supplied by Asahi Chemical were pressed at $290^{\circ} \mathrm{C}$ for $5 \mathrm{~min}$ and allowed to quenching in ice water to obtain a pressed film of $c a .50 \mu \mathrm{m}$ and $200 \mu \mathrm{m}$. The thin one was used for the in-situ birefringence measurement and the thick one was used to prepare quenched samples for X-ray measurement. Thermal treatment was carried out following the scheme shown in Figure 12. The pressed film was heated at $5{ }^{\circ} \mathrm{C} \mathrm{min}^{-1}$ from room temperature and melted at $T_{\max }=270^{\circ} \mathrm{C}$ for $5 \mathrm{~min}$ followed by crystallization at $T_{\mathrm{c}}=250^{\circ} \mathrm{C} . T_{\mathrm{m}}$ was $261^{\circ} \mathrm{C}$.

In-situ birefringence measurement was carried out with a home-built apparatus. A polarizer and an analyzer are set under the crossed polar condition, each making an angle of $45^{\circ}$ with respect to the vertical magnetic field of $6 \mathrm{~T}$. Under this condition, the transmitting light intensity is proportional to $\sin ^{2}(\pi d \Delta n / \lambda)$, where $d$ is the film thickness, $\Delta n$ is the birefringence, and $\lambda$ is the wavelength $(632.8 \mathrm{~nm})$ of the impinging light (He- 


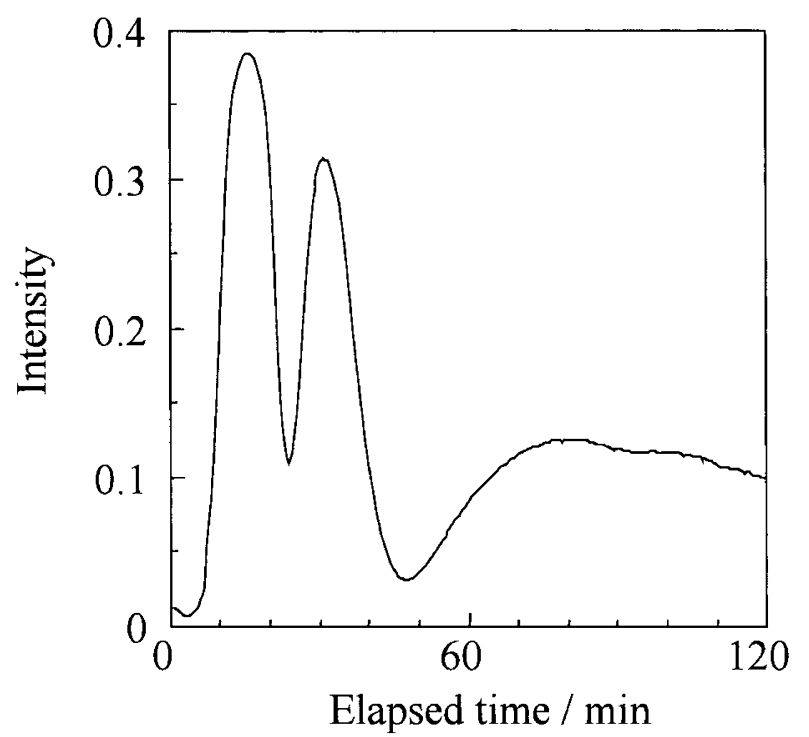

Figure 13. Temporal change in magnetic birefringence of PET sample measured under the crossed polar setting in the field of $6 \mathrm{~T}$. Crystallization temperature was $T_{\mathrm{c}}=250^{\circ} \mathrm{C}$.

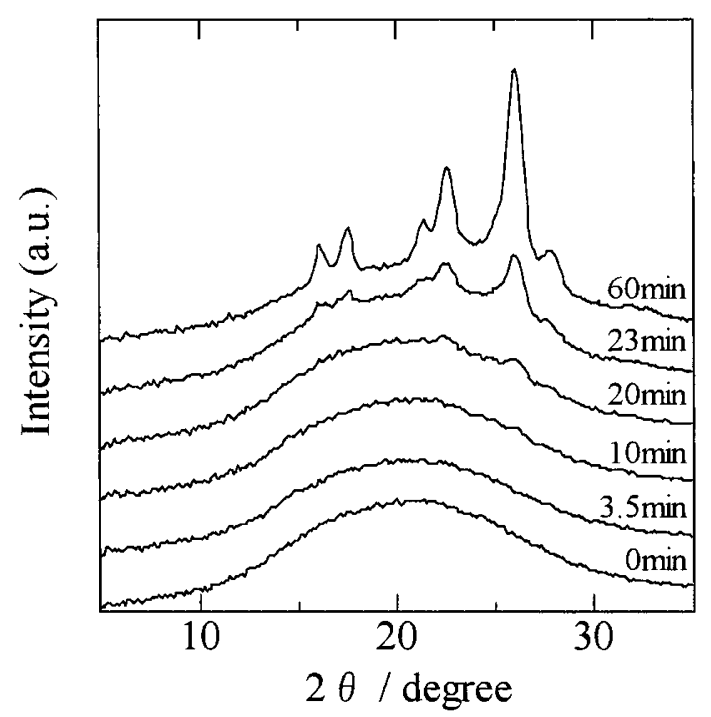

Figure 14. $X$-Ray diffraction patterns for PET samples prepared by quenching at different periods of crystallization time indicated in the figure during the crystallization process same as Figure 13.

Ne laser). The quantity $d \Delta n$ is referred to as retardation. If the increase in $\Delta n$ due to the magnetic alignment of the polymer chain is large, the intensity would even oscillate.

The result of birefringence measurement is shown in Figure 13. The origin of the time axis corresponds to the time at which the temperature reaches the crystallization temperature $T_{\mathrm{c}}\left(250^{\circ} \mathrm{C}\right)$. The oscillation, indicating the alignment, starts at very early stage of crystallization. The first maximum of the transmitting light intensity indicates that the retardation $d \Delta n$ becomes larger than $\lambda / 2$ at this time of crystallization. The wide angle X-Ray diffractions for the samples quenched at

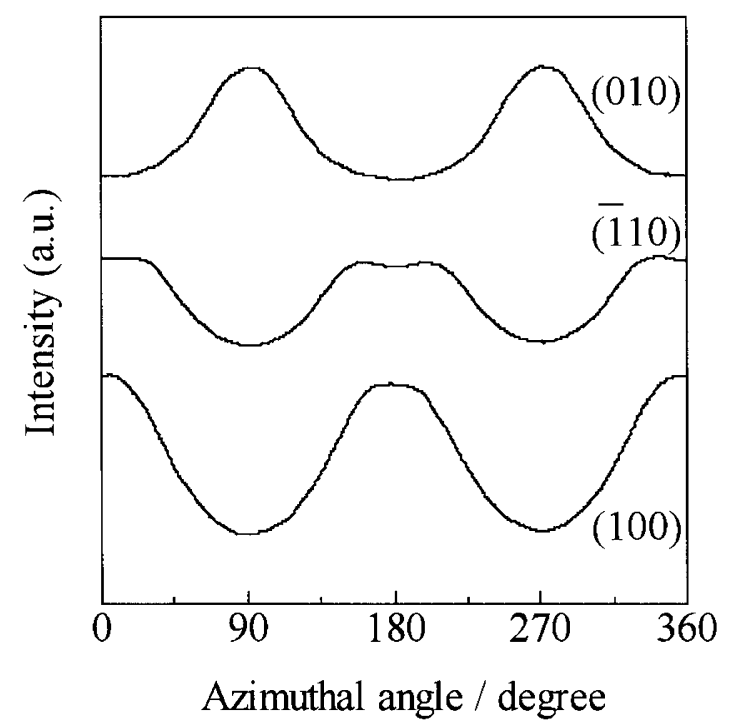

Figure 15. X-Ray azimuthal scans for various planes of PET crystallized from a melt in the magnetic field. Angles $90^{\circ}$ and $270^{\circ}$ correspond to the applied field direction.

various times of crystallization are shown in Figure 14. Up to $20 \mathrm{~min}$, no crystal peaks are observed. Combining this X-Ray result with the birefringence result, we conclude that the origin of the alignment is not the crystal but mesophase. The same conclusion is drawn for PEN $^{91}$ and iPS. ${ }^{92}$

The sample crystallized up to 60 min exhibits crystal peaks. Azimuthal scans of the diffractions for (010), (110), and (100) of this sample are shown in Figure 15. The azimuthal angles of $90^{\circ}$ and $270^{\circ}$ correspond to the direction of the applied field. It is concluded from these diffraction patterns that the $b^{*}$ - and $a^{*}$-axes approximately align parallel and perpendicular to the magnetic field, respectively. This alignment manner is explained by the crystal structure of PET. ${ }^{111}$ In the crystal of PET, the direction of the $a^{*}$-axis lies approximately perpendicular to the plane of the aromatic ring. Therefore, the absolute value of the diamagnetic susceptibility $|\chi|$ is expected to be the largest in the direction of the $a^{*}$-axis due to a ring current induced on an aromatic ring. As a result, the $a^{*}$-axis aligns perpendicular to the magnetic field. Since the PET crystal is triclinic, the $a^{*}$-axis may not exactly coincide with one of the three principal axes of the susceptibility tensor. ${ }^{14}$

The crystal alignment shown above gives us a clue regarding the mesophase structure responsible for the alignment. Assume that the mesophase is nematic-like, that is, the PET chains are somewhat extended, but unlike in the crystal phase they are not fully extended but twisted in a nematic domain. As a result, their aromatic ring planes do not direct in the same direction, but instead they might be distributed randomly along the axis, as schematically illustrated in Figure 16. As a result of 


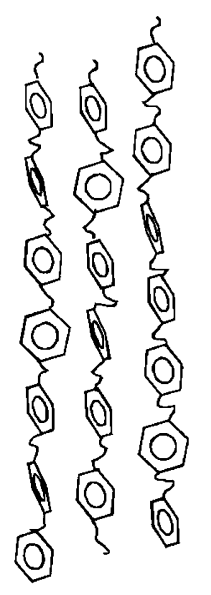

Figure 16. Schematic representation of the PET chain conformation assumed in a nematic-like phase.

this arrangement of the aromatic rings, the $|\chi|$ value of a nematic domain is the largest in the direction perpendicular to the chain axis, leading to the parallel chain alignment with respect to the applied magnetic field. As the crystallization proceeds, the mesophase turns out to be crystal phase, resulting in crystal alignment with the $c$-axis aligned parallel to the applied field. However, this is not what we see in Figure 15. Therefore, we conclude that the mesophase structure responsible for the magnetic alignment is not a nematic-like structure but rather close to the crystal structure.

The above view does not necessarily rule out the possibility of the nematic-like structure formation during the crystallization process. Our observation just means that a mesophase close to the crystal structure happens to be most sensitive to the magnetic field with respect to the size and the anisotorpic susceptibility under the specific thermal history applied in this experiment. The process of structure formation could be different under other thermal histories, so that other types of intermediate structures may be most sensitive to the applied field. In fact, the orientation manner of PEN depends on the thermal history. PEN aligns with the chain axis parallel to the applied field in most cases, but different alignment patterns appear under some other conditions. ${ }^{106}$

Optical properties of PET heat-treated under a magnetic field (6 T) are summarized in Table II. ${ }^{112}$ In addition to the homopolymer used in the above study, a PET sample containing a $3.3 \mathrm{~mol} \%$ cyclohexadimethanol as a comonomer was also used. The retardation values become as large as $\lambda / 4$ to $\lambda / 2$, indicating a possibility of using these films as retardation films. The transparency of the film decreases with the increase in heat treatment time because the crystallization occurs concomitantly. The retardation value is higher for the copolymer film, so is the transparency. This suggests that it will be possible to optimize the anisotropy and transparency of the
Table II. Birefringence values of magnetically aligned PET films. ${ }^{112} \mathrm{~A}$ : homopolymer $[\eta]=1.05, T_{\mathrm{m}}=257^{\circ} \mathrm{C}$; B: copolymer (cyclohexadimethanol $3.3 \mathrm{~mol} \%,[\eta]=0.8, T_{\mathrm{m}}=246^{\circ} \mathrm{C}$

\begin{tabular}{ccccccc}
\hline sample & $\begin{array}{c}T_{\max } \\
\left({ }^{\circ} \mathrm{C}\right)\end{array}$ & $\begin{array}{c}T_{\mathrm{c}} \\
\left({ }^{\circ} \mathrm{C}\right)\end{array}$ & $\begin{array}{c}\text { tc } \\
(\mathrm{min})\end{array}$ & $\begin{array}{c}\text { thickness } \\
(\mu \mathrm{m})\end{array}$ & $\begin{array}{c}d \Delta n \\
(\mathrm{~nm})\end{array}$ & $\Delta n$ \\
\hline & & & 0 & 200 & 0 & 0 \\
PET & 270 & 245 & 10 & 200 & 0 & 0 \\
& & & 20 & 200 & 24.5 & $1.2 \times 10^{-4}$ \\
(A) & & & 30 & 200 & 236 & $1.2 \times 10^{-3}$ \\
\hline & & & 0 & 210 & $1 \sim 2$ & $7 \times 10^{-6}$ \\
PET & 260 & 235 & 5 & 220 & 253 & $1.15 \times 10^{-3}$ \\
(B) & & & 10 & 210 & 376 & $1.79 \times 10^{-3}$ \\
\hline
\end{tabular}

film by means of the molecular design of the primary structure of polymer chain.

\section{Infrared Spectroscopic Analysis of Mesophase}

Fourier transform infrared (FT-IR) spectroscopy is a powerful means for the differentiation of polymer chain conformations in solid states. ${ }^{13-121}$ Because the conformation of the mesophase differs from the crystalline state or the amorphous state, ${ }^{45}$ its formation could be detected by means of in-situ FT-IR measurements. In fact, FT-IR observation of the conformation change of polyethylene that occurs prior to the crystallization is reported. ${ }^{118}$ Also, in-situ FT-IR measurements during crystallization are reported for PEN ${ }^{120}$ and iPS. ${ }^{121}$

In order to monitor the occurrence of mesophase of PEN, we started with identification of the bands specific to this phase. For this purpose, we prepared a few samples obtained under extremely different thermal conditions, that is, one that encourages high crystallinity (crystallization for a prolonged time), and the other that encourages highly amorphous phase (quenching from a melt). The spectra of these samples were subjected to subtraction to each other to identify the bands specific to the pure crystalline phase and the pure amorphous phase. These pure bands were used to decompose the spectra prepared under intermediate conditions between these two extreme conditions. We found that the samples prepared under these intermediate conditions exhibit the bands that are not attributed either to the crystalline or amorphous phase. We took these bands to identify the mesophase.

PEN sample was melted at $300^{\circ} \mathrm{C}$ followed by crystallization at $255^{\circ} \mathrm{C}$. Spectra were taken at various periods of crystallization time starting at the zero crystallization time (defined as the time when the temperature reached $255^{\circ} \mathrm{C}$ ). Subtraction of the spectrum of the zero crystallization time was made to each spectrum so that a typical amorphous band around $1450 \mathrm{~cm}^{-1}$ disappears. We thus obtained a series of spectra that comprise only bands from the crystalline phase and the mesophase. The bands at 1190 and $1178 \mathrm{~cm}^{-1}$, at- 


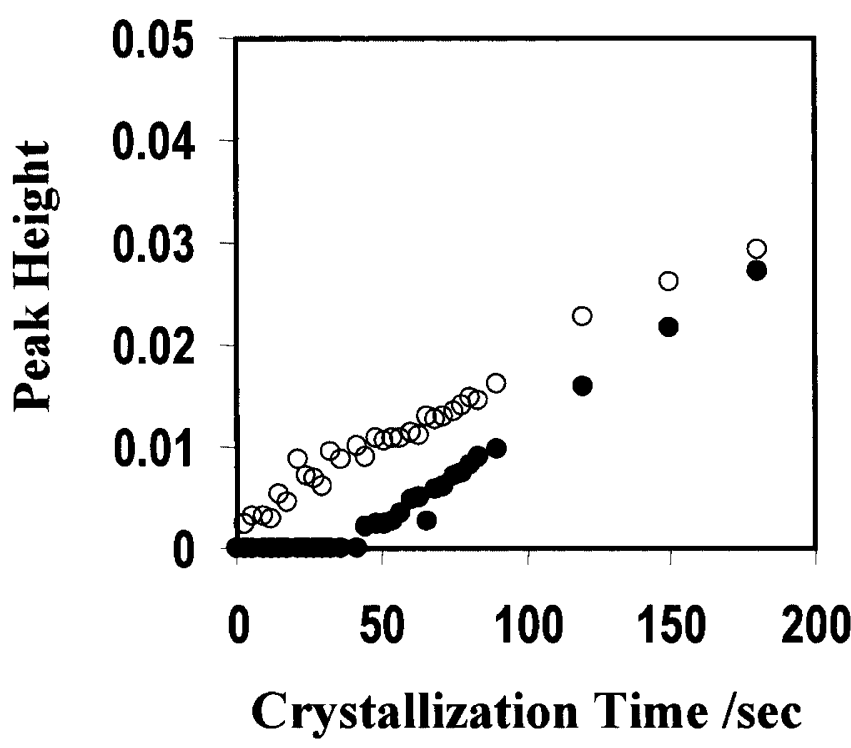

Figure 17. Intensities of the crystal band at $1190 \mathrm{~cm}^{-1}(\bullet)$ and the mesophase band at $1178 \mathrm{~cm}^{-1}(\mathrm{O})$ determined from in-situ FTIR measurement of PEN during crystallization at $255^{\circ} \mathrm{C}$ without magnetic field.

tributed to the crystalline phase and the mesophase, respectively, were used to monitor the increase of the respective phases during crystallization. Figure 17 shows the temporal change of the intensities of these bands. During the initial $40 \mathrm{~min}$, no crystalline formation is observed, while the mesophase formation starts to increase in the very initial stage. This observation supports the scheme that the mesophase is formed prior to the crystal formation.

\section{Magnetic Alignment of "Amorphous" Polymers}

Atactic polymers are classified as amorphous polymers. These polymers literally lack anisotropic structures necessary to undergo magnetic alignment. However, even atactic polymers could have sequences with a relatively high tacticity along the chain, so that these sequences could phase-separate to form anisotropic domains necessary for the magnetic alignment. However, the magnetic alignment of atactic polymers is so far unknown. This is probably because the domain size is too small. If we had polymers whose tacticity is precisely controlled in order to form domains with appropriate sizes, we would be able to attain magnetic alignment of these polymers. Upon stretching, even atactic polymers can form anisotropic domains (elongated regions) that possess anisotropic diamagnetic susceptibility. If this anisotropy remains transiently even upon melting, we may reorient these domains in a desired direction as defined by the field direction. However, we have not succeeded in doing so.

Some polymers crystallize when they are precipitated from a solution, but they do not recover their crystallinity when they are solidified from a melt or cast from a solution with a good solvent. These polymers have a potential of crystallization but used in an amorphous state in many cases. It is difficult to align magnetically these polymers, if we start from a melt or a solution, because they lack a propensity to form anisotropic structures once they loose their crystallinity. For these polymers, an effective means to attain magnetic alignment is to use intermediate states occurring in the course of melting or dissolution where the anisotropy originating from the crystalline state survives in a form of mesophase.

A good example is a magnetic alignment of a cellulose triacetate (CTA) film cast from a solution containing a small amount of a poor solvent. ${ }^{100}$ A crystalline sample of cellulose triacetate (CTA) was dissolved in single solvents (methylene chloride (MC), methyl acetate (MA)) and the mixed solvent of MA with ethanol. Each solution was subjected to the casting under the magnetic field (6 T) and the obtained film was characterized by optical and infrared methods. Only the film cast from the mixed solvent of MA with ethanol $(8: 2 \mathrm{wt} \%$, CTA of $5 \mathrm{wt} \%)$ exhibited birefringence. The value of the birefringence was small $\left(\mathrm{ca} .5 \times 10^{-5}\right)$ because the intrinsic birefringence of CTA is small, but the infrared dichroism measurement revealed that the chain axis is aligned in the direction perpendicular to the applied field. Detailed analyses should be necessary to identify the structure responsible for the magnetic alignment, but we believe that imperfect dissolution of CTA to this mixed solvent and a resultant mesophase is responsible for the alignment. In fact, the solution is opaque, indicating the imperfect dissolution.

Poly(carbonate) (PC) glasses prepared by quenching from a melt hardly develop crystals during cold crystallization process, and hence we hardly expect residual structure remaining after melting. We therefore may not obtain magnetic alignment through this route. However, we know that PC crystallizes when precipitated from a solution. If we start with a precipitate and melt it in the vicinity of the melting point, we might have chance of magnetic alignment. In fact, a magnetic alignment occurs by doing so. ${ }^{122}$ Incidentally, the magnetic alignment of PC is also realized by adding an organic salt reacting and encouraging PC to crystallize. ${ }^{99}$

\section{Alignment of Crystalline Polymers Induced by Mag-} netic Alignment of Nucleating Agent

We have shown that crystalline polymers and socalled amorphous polymers can undergo magnetic alignment under an appropriate condition, but some crystalline polymers do not align. On the other hand, we have also demonstrated that crystallites of low 


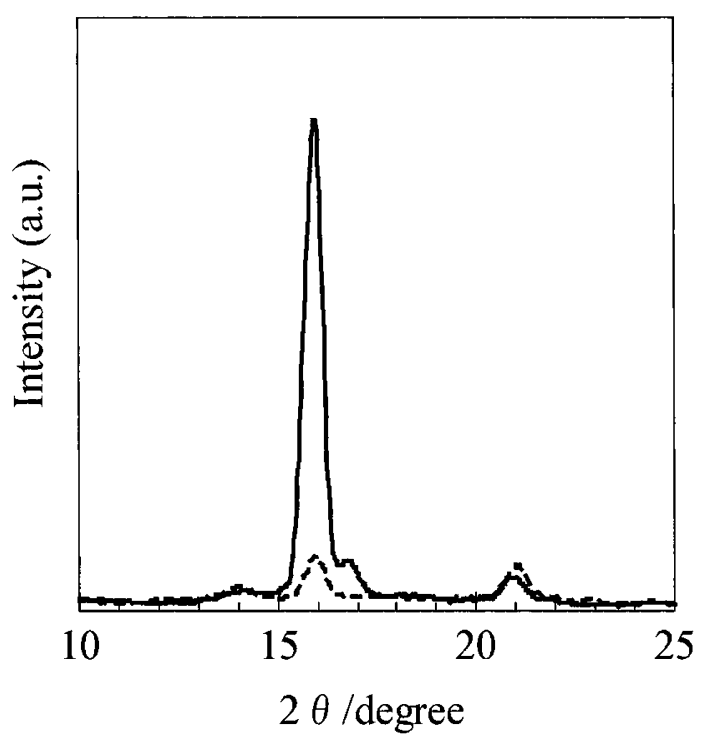

Figure 18. X-Ray diffractions for the $\beta$-form iPP. Solid and broken lines indicate the diffractions in the meridional and equatorial directions, respectively, with the applied magnetic field direction corresponding to the meridional direction. The alignment is induced by the magnetic alignment of DCNDCA crystallites (3\%).

molecular weight compounds suspended in a liquid medium align easily under magnetic fields. Here we demonstrate that the magnetic alignment of a nucleating agent in a polymer melt can induce the alignment of the polymer crystal if the epitaxial crystal growth of the polymer onto the aligned nucleating crystallites occurs.

Crystal of $N, N^{\prime}$-dicyclohexyl-2,6-naphthalenedicarboxamide (DCNDCA), which is reported to encourage the formation of $\beta$-phase iPP, ${ }_{-}^{123}$ was used as a nucleating agent. It aligns with its (104) plane normal to the magnetic field. ${ }^{71}$ Pellets of iPP (Mitsui Chemical JHH grade, $\left.M_{\mathrm{n}}=60000\left(M_{\mathrm{w}}=300000\right)\right)$ were mixed with the DCNDCA crystalline powder (0-3 wt $\%)$ using a mixer at $220^{\circ} \mathrm{C}$ for $5 \mathrm{~min}$. The obtained mixture was hot-pressed at $200^{\circ} \mathrm{C}$ for $10 \mathrm{~min}$, followed by quenching in ice water to obtain a film of $c a .100 \mu \mathrm{m}$ thickness. The thermal treatment of this film was carried out in a home-built furnace placed in a magnetic field of $6 \mathrm{~T}$. The film was heated in the magnetic field at $200^{\circ} \mathrm{C}$ for $5 \mathrm{~min}$, crystallized at $135^{\circ} \mathrm{C}$ for $2 \mathrm{~h}$, and then brought to room temperature to be subjected to the X-Ray analysis.

In the absence of the DCNDCA, the $\alpha$-form crystal was formed. In spite of the application of the external magnetic field, no alignment was observed. On the other hand, the films containing DCNDCA exhibited the alignment of the $\beta$-form crystal when exposed to the field during melt crystallization. The $\mathrm{X}$-Ray diffraction patterns for the (300) of the $\beta$-form iPP shown in Figure 18 clearly show the alignment. Detailed analyses on the X-Ray diffractions for various planes revealed that the iPP $c$-axis aligns perpendicular to the applied field

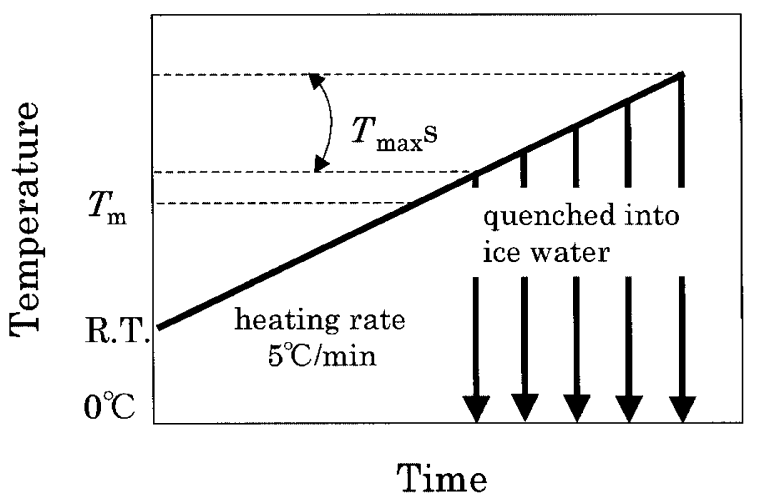

Figure 19. Thermal history applied to the heat-treatment of iPP at $6 \mathrm{~T}$.

and that the (330) plane of the iPP $\beta$-form lies on the $b c$-plane of the DCNDCA crystal in which the direction of the $c$-axis of the iPP coincides with the direction of the $b$-axis of the DCNDCA.

The above technique was also applied successfully to the combination of iPP and $\beta$-phase linear trans-quinacridone, ${ }^{124}$ as well as to the alignment of poly(vinylidene fluoride) ${ }^{125}$ induced by the magnetic alignment of $\beta$-linear trans-quinacridone used as a nucleating agent. We expect that this technique would be useful for other combinations of polymers and nucleating agents, providing a facile means to align crystalline polymers that alone hardly align under magnetic fields.

\section{Melt Structures Viewed by Magnetic Alignment}

Though it is during the induction period of the crystallization that the alignment becomes clear, detailed studies on PET and a low molecular weight iPP (melt flow index of $240 \mathrm{~g} / 10 \mathrm{~min}$ ) have revealed that the origin of the alignment is traced back to their molten states. ${ }^{101}$ Alignment experiments were carried out following a thermal scheme similar to that shown in Figure 12 where the maximum melting temperature $T_{\max }$ is varied. It was found that the magnetic alignment does not occur in the subsequent crystallization process if the $T_{\max }$ is taken high far beyond the melting point $T_{\mathrm{m}}$. In addition, a melt of PET that was exposed to a magnetic field, followed by quenching outside the magnet, became to exhibit alignment upon annealing at cold crystallization temperature outside the magnet. These observations strongly suggest that the residual structures (mesophase) due to the memory effect do play an important role. Furthermore, the mesophase could be traced back to the solid structure before melting.

Figure 19 shows the thermal history used. Samples prepared at different $T_{\max } \mathrm{s}$ were subjected to the optical azimuthal scans to check the alignment. Figure 20 shows the results. In the case of iPP, the samples heated above $260^{\circ} \mathrm{C}$ do not exhibit alignment. Because 


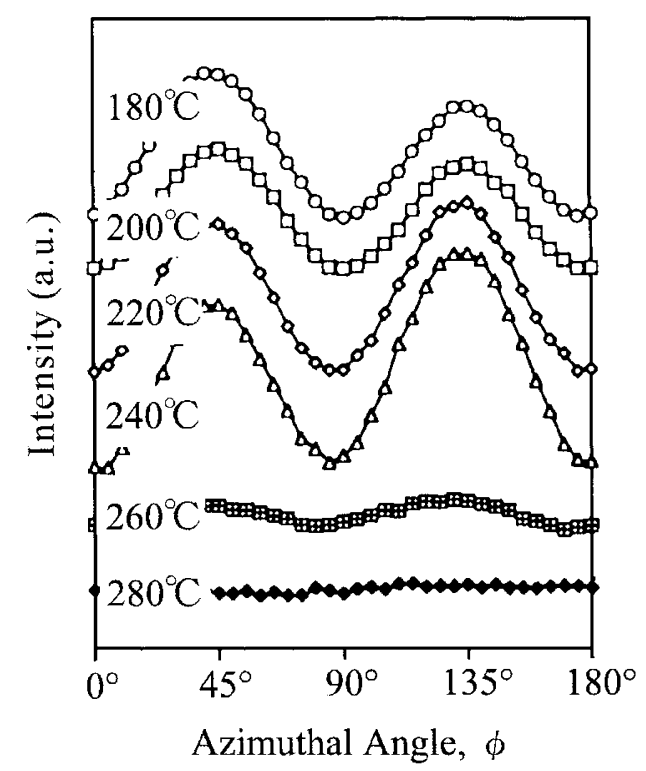

Figure 20. Optical azimuthal scans for iPP samples prepared at $6 \mathrm{~T}$ following the thermal history shown in Figure 19.

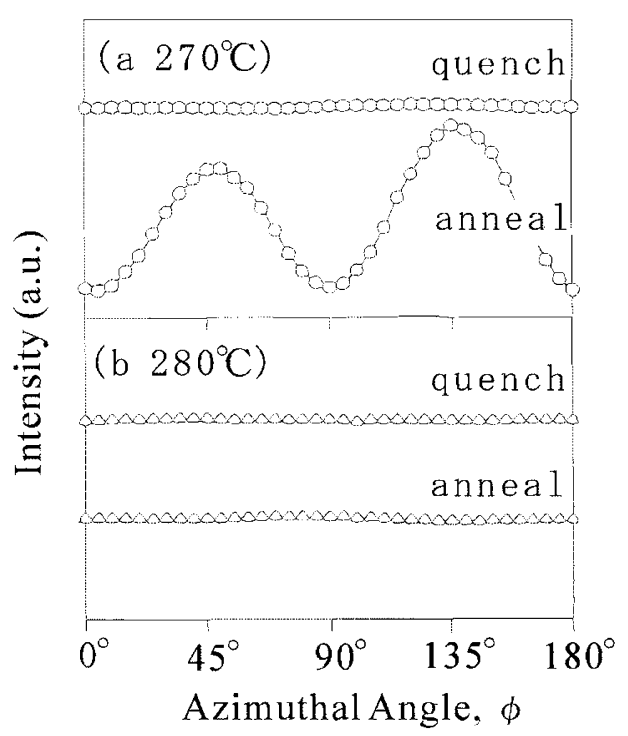

Figure 21. Optical azimuthal scans for PET samples heated up to (a) $T_{\max }=270^{\circ} \mathrm{C}$ and (b) $T_{\max }=280^{\circ} \mathrm{C}$. "quench" indicates asquenched sample and 'anneal' indicates that the quenched sample was annealed at cold crystallization temperature.

the quenching was carried out outside the magnet, the alignment observed for 180 to $240^{\circ} \mathrm{C}$ is attributed to the mesophase existing in the melt, which acts as nuclei causing the crystal alignment. The quenching similar to that for iPP was also carried out for PET. Figure 21 shows the optical azimuthal scans. The alignment was not detectable for the as-quenched samples irrespective of the melting temperature, but upon annealing the sample quenched from a melt at $270^{\circ} \mathrm{C}$ exhibited alignment. This observation leads to the same interpretation as was made for iPP.

The stability of the mesophase in the melt was examined by subjecting the melt to various durations of heat- ing at given $T_{\max } \mathrm{S}$. In the case of iPP, the mesophase was stable by heating at $180^{\circ} \mathrm{C}$ up to $60 \mathrm{~min}$ or more, while it disappeared in $30 \mathrm{~min}$ when heated at $240^{\circ} \mathrm{C}$. In the case of PET, the mesophase remained stable at $270^{\circ} \mathrm{C}$ at least up to $60 \mathrm{~min}$, while no mesophase survived at $280^{\circ} \mathrm{C}$. The mesophase seems stable at temperatures in the vicinity of the melting point $T_{\mathrm{m}}$, but we cannot say whether it is a thermodynamically defined phase or not.

The critical temperature above which the mesophase becomes more or less unstable is easily determined by examining the crystallization behavior using DSC. The onset temperature of the crystallization in the cooling process from a melt depends strongly on at what temperature $T_{\max }$ the sample was melted. If the sample is heated at $T_{\max }$ higher than a critical value, the onset of crystallization shifts significantly to lower temperatures, indicating the loss of mesophase acting as heterogeneous nuclei. Mesophase of iPP above the melting point is also studied by viscoelastic method. ${ }^{126-128}$

The above observation that the thermal history before crystallization affects the subsequent crystallization process is not a peculiar one to the polymer meltcrystallization. Similar observations are reported for low molecular weight compounds undergoing crystallization from solutions. ${ }^{129}$ The temperature limit of a stable supersaturation of a solution becomes significantly low if the solution is superheated before it is cooled to temperatures below the saturation temperature. This observation is explained in terms of clusters existing in the solution. At temperatures just above the saturation temperature it is assumed that some solute molecules are in a form of clusters in the solution. Upon further heating (superheating), these clusters are destroyed so that the nucleation becomes scarce when the solution is cooled down below the saturation temperature, resulting in a stable supersaturation. In the case of crystallization of low molecular weight compounds, one of concerns is to form a large single crystal. For this purpose, the superheating is a good procedure because it reduces the nucleus formation. On the other hand, in the case of the magnetic alignment of crystalline polymers, the use of residual mesophase (possibly an equivalence of cluster) is important, and hence the "superheating" should be avoided.

\section{Non-Rotation Type Alignment - Preferential Meso- phase Formation and Growth-}

Like the pressure effect on the phase transition temperature, the magnetic field can cause a shift $\delta T$ of the phase transition temperature. This shift occurs due to two factors. One is the difference in magnetic susceptibility between two phases, for example, liquid and va- 
por phases. If the molar diamagnetic susceptibilities of these two phases are different, the transition temperature will shift. ${ }^{8}$ Another factor becomes important when one of the relevant phases exhibits anisotropy ${ }^{130}$ such as a crystal phase. ${ }^{107}$ Due to the anisotropy, a crystal with $\chi_{a}>0$, if aligned parallel to the applied field, has a lower magnetic energy than aligned perpendicular to the field. As a result, the melting point of the crystal with parallel alignment becomes higher than that with perpendicular alignment. Then, if a melt is brought down to the temperature between melting points corresponding to these two extreme alignments, crystals with higher melting point (crystals of parallel alignment in this case) are formed preferentially. If randomly oriented crystalline powder is heated at the temperature mentioned above, those which happen to be oriented perpendicular to the field are melted preferentially. This mechanism indicates a possibility of alignment other than the mechanism of domain rotation.

The shift $\delta T$ could affect not only the melting point itself as discussed above, but also the rates of nucleation and growth of the crystallization. These rates are the function of the supercooling, i.e., the temperature difference between the equilibrium melting point and the temperature to which the sample is quenched for crystallization. Because the supercooling is larger for the phase with higher melting point, a preferential nucleation and growth of this phase is expected. This would results in alignment.

Now, let us estimate the magnitude of the shift. Using a derivation similar to that used for the Clapeyron equation, we obtain for the shift value: ${ }^{107}$

$$
\delta T=\frac{\left\{\left(\cos ^{2} \theta-1 / 3\right) \chi_{a}+<\chi_{s}>-\chi_{l}\right\} B^{2}}{2 \mu_{0} \Delta \bar{H}} T_{m},
$$

where $\Delta \bar{H}$ is the molar latent heat of transition, $\chi_{a}=$ $\chi_{/ /}-\chi_{\perp},<\chi_{s}>=\left(2 \chi_{\perp}+\chi_{/ /}\right) / 3$, and $T_{\mathrm{m}}$ is the melting point in the absence of the applied field. The three molar diamagnetic susceptibilities of trans azobenzene are $\chi_{1}=-110 \times 10^{-11}, \chi_{2}=-164 \times 10^{-11}, \chi_{3}=$ $-104 \times 10^{-11} \mathrm{~m}^{3} \mathrm{~mol}^{-1} 131$ in SI units. The molar enthalpy of melting is $\Delta \bar{H}=22 \times 10^{3} \mathrm{~J} \mathrm{~mol}^{-1}$ and the melting point is $T_{\mathrm{m}}=342 \mathrm{~K}$. Assuming an axial symmetry around $\chi_{2}$, we obtain $\chi_{a}=-6 \times 10^{-10} \mathrm{~m}^{3} \mathrm{~mol}^{-1}$. Then, the shift is calculated to be $\delta T=2.5 \times 10^{-4} \mathrm{~K}$ when the magnetic field of $10 \mathrm{~T}$ is applied to the direction perpendicular to the $\chi_{2}$ axis. Here, $\left\langle\chi_{s}\right\rangle=\chi_{l}$ is assumed. The nematic-isotropic transition of $p$ azoxyanisole (PAA) exhibits a much larger shift. The shift is about $\delta T=5 \mathrm{mK}$. This large shift is attributed mainly to the small value of $\Delta \bar{H}=0.574 \times 10^{3} \mathrm{~J} \mathrm{~mol}^{-1}$.

This magnitude of the shift yet seems to be small to expect a preferential alignment to occur. However, recent work about thermal analysis in a strong magnetic field ${ }^{108,109}$ has revealed that the shifts observed for paraffin and some other organic compounds are several orders higher than the calculated values. These experimental results could indicate that the crystal formation involves the mesophase formations that only need small transition enthalpies. We cannot rule out the possibility of preferential alignment. The detail will be reported elsewhere. ${ }^{132}$

\section{MAGNETIC LEVITATION}

\section{General View}

In the previous sections, we considered spatially homogeneous magnetic fields applied to magnetically anisotropic particles or phases to induce the alignment. We now turn to the use of inhomogeneous magnetic fields applied, for simplicity, to a magnetically isotropic particle. Here, the effect of the gravity also should come into consideration. Now, the energy that a particle has is expressed as

$$
E=-(1 / 2) V \chi \mu_{0}^{-1} B^{2}(x, y, z)+m g z,
$$

where $V$ and $m$ is the volume and the weight of the particle, respectively, $\mu_{0}$ is the magnetic permeability of the vacuum, $\chi$ is the volumetric diamagnetic susceptibility of the particle, $z$ is the vertical coordinate, and $g$ is the gravitational acceleration. If the field is spatially inhomogeneous, the magnetic energy of a particle (the first term) in eq 13 is a function of the coordinates. The force acting on the particle is given by $F=-\operatorname{grad} E$. In a simple case that $B$ is a function of only $z$, we obtain

$$
F=V \chi \mu_{0}^{-1} B d B / d z+m g .
$$

If the magnetic and gravitational forces balance with each other, then we obtain

$$
-\rho g+\chi \mu_{0}^{-1} B d B / d z=0,
$$

where $\rho$ is the density of the particle. Unlike the buoyancy, the gravitational force is compensated by the magnetic force at the atomic level. A water droplet, for example, levitates if $B d B / d z=1400 \mathrm{~T}^{2} / \mathrm{m}$. A few groups have succeeded to levitate diamagnetic materials including a water droplet, a strawberry, a tomato, a rice grain, and even a frog and a grasshopper. ${ }^{1-3}$

In the above equations, the effect of the medium surrounding the particle is ignored. To be rigorous, the hydrodynamic and magnetic buoyancies exerted by the surrounding medium should be included. For this purpose, eq 15 is modified to

$$
-\Delta \rho g+\Delta \chi \mu_{0}^{-1} B d B / d z=0,
$$

where $\Delta \rho=\rho_{1}-\rho_{2}$ and $\Delta \chi=\chi_{1}-\chi_{2}$, with suffices 1 and 2 indicating the particle and the surrounding 


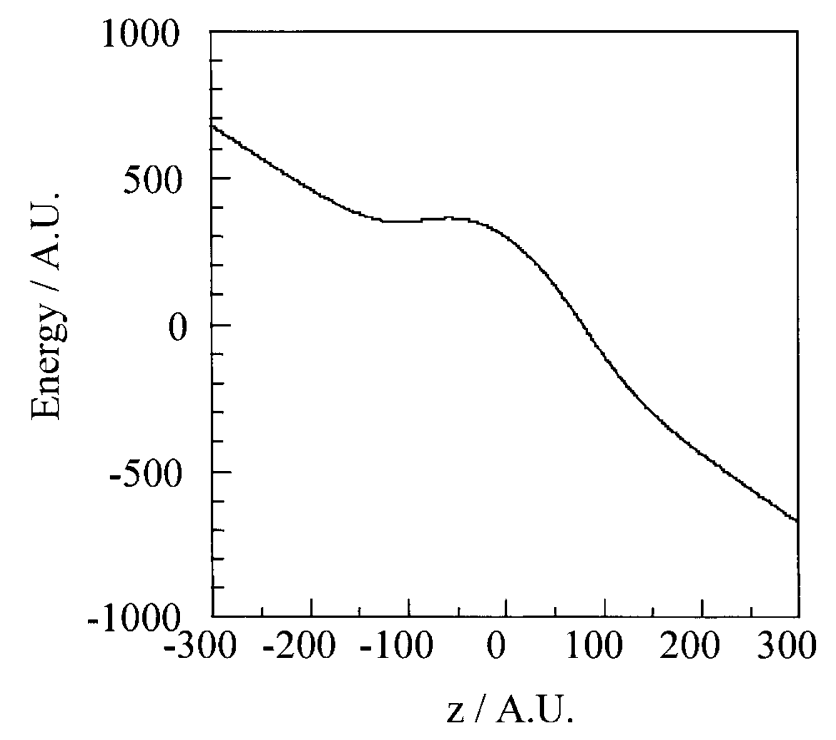

Figure 22. The energy $E$ in eq 13 is schematically plotted as a function of the vertical coordinate $z$. Depending on the profile of $B$, and the parameters $m, V$, and $\chi$, a minimum is formed, where a particle is trapped.

medium, respectively. The contributions by $\rho_{2}$ and $\chi_{2}$ are from the hydrodynamic and magnetic buoyancies, respectively. Both of them act on the particle through the surface. This is in contrast to the contributions by $\rho_{1}$ and $\chi_{1}$ which act at the atomic level. In the vacuum, eq 16 reduces to eq 15 because $\rho_{2}$ and $\chi_{2}$ vanish. In the air, eq 15 is a good approximation because $\Delta \rho \cong \rho_{1}$ and $\Delta \chi \cong \chi_{1}$. Because $B d B / d z=g \mu_{0} \Delta \rho / \Delta \chi$, the effective way to make a particle levitate under a moderated magnetic condition is to diminish the value of $\Delta \rho / \Delta \chi$. This can be done if we use a paramagnetic fluid as a surrounding medium (Magneto-Archimedes levitation). ${ }^{133}$

A remarkable feature of the diamagnetic levitation is its trapping effect, which is in contrast to the levitation realized by hydrodynamic buoyancy or the levitation in the space. With an appropriate choice of the field profile, the energy expressed in eq 13 exhibits a minimum, as is schematically demonstrated in Figure 22, indicating that the particle is trapped at this vertical point. At the same time, the trapping is possible with respect to the horizontal directions. The detail of the stability condition is reported by Berry and Geim. ${ }^{3}$

The magnetic force could also balance with the entropic force. The equation of balance is described by

$$
\left(\frac{\partial \mu}{\partial x}\right)=\mu_{0}^{-1} \chi V B\left(\frac{\partial B}{\partial x}\right) N_{\mathrm{A}},
$$

where the left-hand side corresponds to the entropic force with $\mu$ being chemical potential. $N_{\mathrm{A}}$ and $V$ are Avogadro's constant and the volume of a solute particle or a molecule under consideration, respectively. The chemical potential is related to the concentration as

$$
\mu=\mu^{*}+R T \ln \gamma c,
$$

where $\gamma$ is the activity coefficient and $\mu^{*}$ is the chemical potential at a standard state. Using this equation, eq 17 is rewritten as

$$
k_{\mathrm{B}} T\left(1+\frac{\partial \ln \gamma}{\partial \ln c}\right)\left(\frac{\partial c}{\partial x}\right)=c \mu_{0}^{-1} \chi V B\left(\frac{\partial B}{\partial x}\right) .
$$

The quantity $1+(\partial \ln \gamma) /(\partial \ln c) \equiv f$ in the above equation is unity for an ideal solution, but could be smaller than unity for non-ideal solutions. If we suppose that $f$ is constant, then eq 19 is integrated to be

$$
c(x)=c_{0} \exp \left(\frac{\mu_{0}^{-1} \chi V B^{2}(x)}{2 f k_{\mathrm{B}} T}\right),
$$

where $c=c_{0}$ and $B=0$ at $x=x_{0}$ is assumed. We see that if the factor $f$ is small and/or the volume $V$ of the solute particle is large, the magnetic field $B(x)$ can cause a significant concentration profile. The effect of a suspending liquid or a solvent can be accounted for just by putting $\chi \rightarrow \Delta \chi \equiv \chi_{1}-\chi_{2}$ with suffices 1 and 2 indicating the particle and the surrounding medium, respectively.

\section{Magnetic Separation of Polymers}

Depending on their values of $\Delta \rho / \Delta \chi$, particles levitate at different vertical locations. This indicates that the separation is possible. In fact, it is reported that a mixture of sugar and salt, ${ }^{134}$ a mixture of colored glass grains, ${ }^{135}$ etc. are separated into the constituent components. In this section, we demonstrate the separation of solid polymers suspended in a paramagnetic aqueous solution, using Magneto-Archimedes levitation. ${ }^{136}$

Polymer samples used are pellets of atactic polystyrene (PS), poly(ethylene terephthalate) (PET), poly(methyl methacrylate) (PMMA), syndiotactic polypropylene (sPP), and styrene-butadiene block copolymer (SB). An aqueous solution of manganese chloride $\left(\mathrm{MnCl}_{2}\right)$ with $\rho_{2}=1.098 \mathrm{~g} \mathrm{~cm}^{-3}$ and $\chi_{2}=$ $1.7 \times 10^{-4}$ was used for a suspending medium. The density of the solution is lower than those of PET and PMMA and larger than those of the others. An electromagnet generating the field profile $B(z)$ and the profile of $B d B / d z$ (Figures 23 (a), (b)) was used. Above the center $(z=0)$ of the two pole pieces, an upward force acts on the pellets, while the force is downward below the center. The pellets were put in a test tube and the solution was poured. PET and PMMA pellets sink in the bottom of the test tube, while the other pellets float on the surface of the liquid. Figure 24 shows the photos of levitation. PET and PMMA pellets levitate when they are put above the field center. The others are pushed into the solution (anti-levitation) when they are put below the field center. Each pellet is located in different vertical places. 


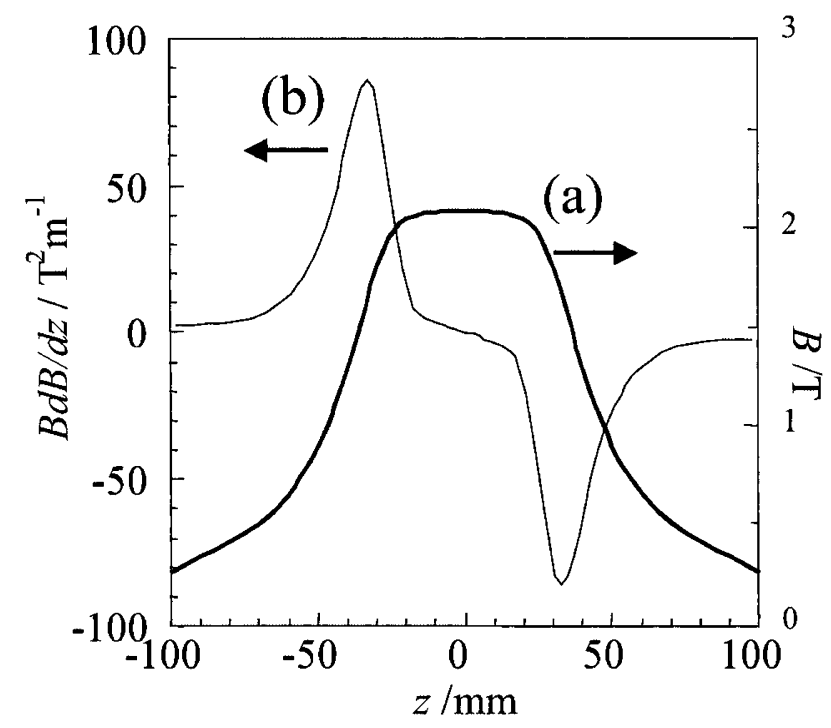

Figure 23. Magnetic field profile $B(z)$ (a), and the quantity $B d B / d z$ (b) generated by an electromagnet.

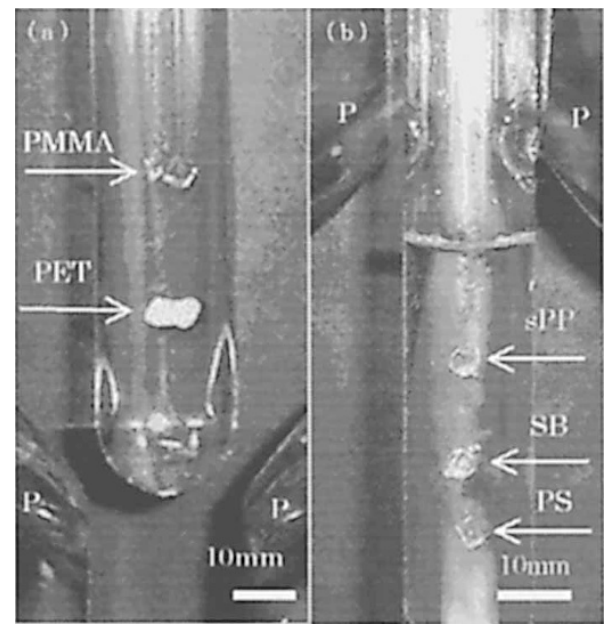

Figure 24. Magneto-Archimedes levitation of (a) PET and PMMA (poly(methyl methacrylate)) and (b) anti-levitation of sPP (syndiotactic polypropylene), SB (styrene-butadiene copolymer), and PS (atactic polystyrene).

\section{Levitation Polymerization}

Polymer spheres are fabricated using suspension and emulsion polymerization. However, the size of the spheres is limited to the order of micrometers or less because a stable suspension of monomer droplets is difficult if their size becomes large. An advantage of the use of magnetic levitation is that larger droplets (the order of millimeters or even centimeters) can be levitated in a stable manner. A droplet is trapped at the same location throughout the whole process of polymerization without sinking to the bottom or wondering against the container wall to result in distortion of spherical shape. The density of the droplet could change during polymerization, and so does the location of the levitation. However, it is possible that the levitation point is maintained at the same location by changing the current sup-

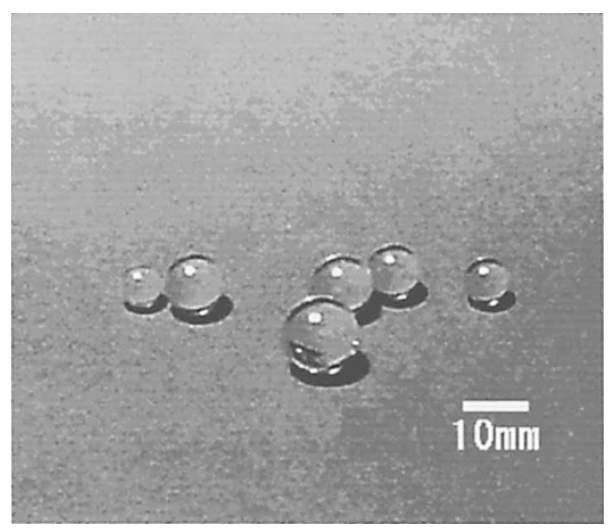

Figure 25. Large poly(benzyl methacrylate) spheres fabricated by levitation polymerization. Size is about $7-9 \mathrm{~mm}$ in diameter.

ply to the magnet.

Figure 25 shows poly(benzyl methacrylate) spheres fabricated by means of "Levitation Polymerization". ${ }^{137}$ A ca. $1 \mathrm{~mL}$ benzyl methacrylate (containing $50 \mathrm{ppm}$ inhibitor) with $0.7 \%$ benzoyl peroxide added as an initiator was slowly injected into the aqueous solution of $\mathrm{MnCl}_{2}$ at $70^{\circ} \mathrm{C}$ with a pipet. The droplet formed was trapped below the center of a vertical field generated by a superconducting magnet. Namely, the experiment was carried out at anti-levitation mode (the droplet is pushed downward). As polymerization proceeds, the droplet increased its density to start sinking. To prevent sinking, the field strength was reduced from $1.4 \mathrm{~T}$ to $c a$. $1.0 \mathrm{~T}$ (referred to at the center) around $60 \mathrm{~min}$ after the injection. As a result, the trapping location was maintained at the same place. After $5 \mathrm{~h}$ polymerization in the magnet, the droplet was taken out from the magnet and further polymerized in boiling water outside the magnet. The size of the fabricated spheres is $c a$. $7-9 \mathrm{~mm}$ in diameter. The average deviation with respect to the average diameter was about $0.6 \%$.

\section{CONCLUSIONS}

Magnetic effects on "non-magnetic" materials are small but existing in any materials including polymeric materials. They are too small to be detected easily under the field strengths as low as generated by permanent magnets and electromagnets. Advent of liquid-helium free superconducting magnets has facilitated the use of high magnetic fields to result a number of new findings that would have been impossible under low fields. The use of high fields has been paid attention in various fields in academia and industries.

Crystalline polymers were considered to be unable to undergo magnetic alignment because they lack ordered structures required for the alignment to occur. However, we have found that a number of 
crystalline polymers including poly(ethylene terephthalate), poly(ethylene-2,6-naphthalate), etc. do undergo magnetic alignment during crystallization from melts. The origin of the alignment is attributed to anisotropic structures (mesophase) transiently forming during crystallization and/or existing in the melt. Current understanding of the alignment mechanism is that these anisotropic structures rotate under magnetic torque, resulting alignment. Another possibility of preferential formation and preferential growth of anisotropic structures in a specific direction with respect to the field, resulting in alignment, is not ruled out. Since the polymeric systems are rich in mesophase especially during phase transition, many magnetic effects are expected if polymeric systems are exposed to the external magnetic field during they are undergoing a phase transition.

The use of moderate field strengths may be preferred in the actual application of the magnetic alignment in industries. This is possible in suspensions. Fibers and fine crystalline particles of sub-micron sizes suspended in a low viscosity liquid can highly and quickly align in moderate fields provided by electromagnets or even permanent magnets. The key issue here is to disperse these particles in a stable manner and to fix the attained alignment. Precision alignment, which enables uniaxial alignment of negative anisotropic $\left(\chi_{a}<0\right)$ fibers, for example polyethylene fiber, would be useful to attain desired alignment of crystallites and etc.

Levitation is another field of interest. Pseudo-zerogravitation circumstances are provided by the magnetic levitation. They are different from the zero-gravity realized in the space in that the effect of trapping and alignment also comes in. In addition, if the medium surrounding the levitating particle is relevant, the hydrodynamic and magnetic buoyancies become dominant, which deviates the levitation from the pure zero-gravity circumstances. The magnetic force causing levitation would be also useful for micro- to nano-patterning of particles, if the microscopic inhomogeneity of magnetic field is available.

Superconducting technology is continuously progressing. In future, 30 -tesla class magnets may become widely accessible to individual laboratory level like 10tesla ones at present time. Further progress is expected in future in academia as well as in industry.

Acknowledgment. The author thanks Prof. Y. Morishima, Editor-in-Chief, for inviting to this review article. The author thanks Prof. E. Ito for continuous encouragement. Discussion with Drs. M. Yamato, H. Sata, H. Ezure, S. Kossikhina, T. Kawai, and F. Kimura is greatly appreciated. This work has been partially supported by Japan Society for the Promotion of Sci- ence through Research for the Future Program.

\section{REFERENCES}

1. See for example,

a) Annual Reports of Tsukuba Magnet Laboratory, National Institute for Materials Science.

b) Annual Reports of High Field Laboratory for Superconducting Materials, Institute for Materials Research, Tohoku University.

c) K. Watanabe, Y. Yamada, J. Sakuraba, F. Hata, and C. K. Chong, Jpn. J. Appl. Phys., 32, L488 (1993).

2. E. Beaugnon and R. Tournier, Nature, 349, 470 (1991).

3. M. V. Berry and A. K. Geim, Eur. J. Phys., 18, 307 (1997).

4. N. Kitamura, M. Makihara, M. Hamai, T. Sato, I. Mogi, S. Awaji, K. Watanabe, and M. Motokawa, Jpn. J. Appl. Phys., 39, L324 (2000).

5. N. Hirota, T. Homma, H. Sugawara, K. Kitazawa, M. Iwasaka, S. Ueno, H. Yokoi, Y. Kakudate, S. Fujiwara and K. Kawamura, Jpn. J. Appl. Phys. 34, L991 (1995).

6. "Materials Science in Static High Magnetic Fields", K. Watanabe and M. Motokawa Eds., Springer Verlag, Berlin (2001).

7. a) "Proc. of The 3rd Meeting of Symposium on New Magneto-Science'99”, Omiya, November, 1999.

b) "Proc. of The 6th Meeting of Symposium on New Magneto-Science'02", Tsukuba, November, 2002.

8. "Jikikagaku", (in Japanese) K. Kitazawa, S. Ozeki, S. Tanimoto, and M. Yamaguchi Eds., IPC, Tokyo (2002).

9. G. Maret and K. Dransfeld, in "Topics in Applied Physics", F. Herlach Ed., Springer Verlag, Berlin, 1985, Vol. 57, Chapt. 4.

10. S. Nagakura, H. Hayashi, and T. Azumi, "Dynamic Spin Chemistry”, (Kodansha; Wiley, Tokyo, New York, 1998).

11. "Riken Review", No. 44 (2002).

12. Y. Tanimoto, H. Hayashi, S. Nagakura, H. Sakuragi, and K. Tokumaru, Chem. Phys. Lett., 41, 267 (1976).

13. J. H. van Vleck, "The Theory of Electric and Magnetic Susceptibilities", Oxford University Press, London, 1932.

14. A. Weiss and H. Witte, "Magnetochemie", Verlag Chemie GmbH, Weinheim, 1973 (Japanese translation).

15. M. Fujiwara, T. Chidiwa, R. Tokunaga, and Y. Tanimoto, $J$. Phys. Chem. B, 1023417 (1998).

16. M. Fujiwara, M. Fukui, and Y. Tanimoto, J. Phys. Chem. B, 103, 2627 (1999).

17. G. Sazaki, E. Yoshida, H. Komatsu, T. Nakada, S. Miyashita, and K. Watanabe, J. Cryst. Growth, 173, 231 (1997).

18. N. I. Wakayama, M. Ataka, and H. Abe, J. Cryst. Growth, 178, 653 (1997).

19. N. I. Wakayama, Cryst. Growth Des., 3, 17 (2003).

20. V. Timbrell, J. Appl. Phys., 43, 4839 (1972).

21. Y. Schmitt, C. Paulick, F. X. Royer, and J. G. Gasser, J. NonCryst. Solids, 205-207, 139 (1996).

22. M. Fujiwara, E. Oki, M. Hamada, Y. Tanimoto, I. Mukouda, and Y. Shimomura, J. Phys. Chem. A, 105, 4383 (2001).

23. T. Kimura, H. Ago, M. Tobita, S. Ohshima, M. Kyotani, and M. Yumura, Adv. Mater, 14, 1380 (2002).

24. M. J. Casavant, D. A. Walters, J. J. Schmidt, and R. E. Smal- 
ley, J. Appl. Phys., 93, 2153 (2003).

25. J. Sugiyama, H. Chanzy, and G. Maret, Macromolecules, 25, 4232 (1992).

26. J.-F. Revol, L. Godbout, X. M. Dong, D. G. Gray, H. Chanzy, and G. Maret, Liq. Cryst., 16, 127 (1994).

27. X. M. Dong and D. G. Gray, Langmuir, 11, 3029 (1997).

28. Y. Kawamura, I. Sakurai, A. Ikegami, and S. Iwayanagi, Mol. Cryst. Liq. Cryst., 67, 77 (1981).

29. J. Torbet, J.-M. Freyssinet, and G. Hudry-Clergeon, Nature, 289, 91 (1981)

30. A. Yamagishi, T. Takeuchi, T. Higashi, and M. Date, Physica $B, \mathbf{1 7 7}, 523$ (1992).

31. M. Murayama, Nature, 206, 420 (1965).

32. J. Torbet and M. Y. Norton, FEBS Lett., 147, 201 (1982).

33. M. Fujiwara, R. Tokunaga, and Y. Tanimoto, J. Phys. Chem. $B, \mathbf{1 0 2}, 5996$ (1998).

34. A. Makiya, D. Shouji, S. Tanaka, N. Uchida, T. Kimura, and D. Uematsu, Key Eng. Mat., 206-213, 445 (2002).

35. H. Morikawa, K. Sassa, and S. Asai, Mat. Trans. JIM, 39, 8 (1998).

36. T. Taniguchi, K. Sassa, T. Yamada, and S. Asai, Mat. Trans. $J I M, 41,981$ (2000).

37. T. S. Suzuki, Y. Sakka, and K. Kitazawa, Adv. Eng. Mater., 3, 163 (2001).

38. R. R. Gupta, "Landolt-Bornstein", H.-H Hellwege and A. W. Hellwege Eds., Springer Verlag, Berlin, 1986, vol.II/16, Chapt.8.

39. "Polymer Crystallization", Lecture Notes in Physics 606, G. Reiter and J.-U. Sommer Eds., Springer Verlag, Berlin, 2003; Proc. of 263. WE-Heraeus-Seminar, Waldau, Germany, October, 2001.

40. "Proc. of International Symposium on Polymer Crystallization”, Mishima, Japan, June, 2002.

41. G. R. Strobl, "The Physics of Polymers", Springer Verlag, Berlin, 1996.

42. G. R. Strobl, Eur. Phys. J. E, 3, 165 (2000).

43. P. D. Olmsted, W. C. K. Poon, T. C. B. McLeish, N. J. Terrill, and A. J. Ryan, Phys. Rev. Lett., 81, 373 (1998).

44. M. Imai, K. Mori, T. Mizukami, K. Kaji, and T. Kanaya, Polymer, 33, 4451 (1992).

45. M. Imai, K. Kaji, and T. Kanaya, Phys. Rev. Lett., 71, 4162 (1992).

46. M. Muthukumar and P. Welch, Polymer, 41, 8833 (2000).

47. Y. A. Akpalu, E. J. Amis, J. Chem. Phys., 111, 8686 (1999).

48. Y. A. Akpalu, E. J. Amis, J. Chem. Phys., 113, 392 (2000).

49. K. Kaji, "Handbook of Thermoplastic Polyesters", S. Fakirov, Ed. Chapt. 4, Wiley-VCH, Weinheim, 2002.

50. H. Morioka, T. Kimura, and R. Aogaki, Trans. MRS-J., 27, 129 (2002).

51. H. Morioka, T. Kimura, and R. Aogaki, Chem. Lett., 2002, 962.

52. I. Mogi, K. Watanabe, and M. Motokawa, Electrochemistry, 67, 1051 (1999).

53. I. Mogi, K. Watanabe, and M. Motokawa, J. Electroanal. Chem., 507, 198 (2001).

54. Y. Matsumoto, I. Yamamoto, Y. Shimazu, M. Yamaguchi, and F. Ishikawa, Jpn. J. Appl. Phys., 36, L1397 (1997).

55. I. Yamamoto, Y. Matsumoto, M. Yamaguchi, Y. Shimazu, and F. Ishikawa, Physica B, 246-247, 408 (1998).
56. K. Akagi, M. Narita, R. Toyoshima, and H. Shirakawa, Mol. Cryst. Liq. Cryst., 318, 157 (1998).

57. N. Hirota, Y. Ikezoe, H. Uetake, T. Kaihatsu, T. Takayama, and K. Kitazawa, MRS-J, 27, 47 (2002).

58. T. Kimura, S. Mamada, and M. Yamato, Chem. Lett., 2000, 1294.

59. A. Nara, M. Yamato, and T. Kimura, "Proc. of The 6th Meeting of Symposium on New Magneto-Science'02”, Tsukuba, November, p. 153, 2002.

60. T. Kimura, M. Yamato, W. Koshimizu, M. Koike, and T. Kawai, Langmuir, 16, 858 (2000).

61. M. Doi, and S. F. Edwards, Theory of Polymer Dynamics; Clarendon Press, Oxford, 1986, Chapt. 8 and references therein.

62. G. B. Jeffery, Proc. R. Soc. London, Ser. A, 102, 161 (1992).

63. F. Perrin, J. Phys. Radium., 5, 497 (1934).

64. M. Yamato, H. Aoki, T. Kimura, I. Yamamoto, F. Ishikawa, M. Yamaguchi, and M. Tobita, Jpn. J. Appl. Phys., 40, 2237 (2001).

65. C. B. Scott and D. B. Fischbach, J. Appl. Phys., 47, 5329, (1976).

66. H. Aoki, T. Kimura, and M. Tobita, Polym. Prepr. Jpn., 51, 3753 (2002).

67. T. Kimura, N. Shimoyama, and M. Tobita, Polym. Prepr. Jpn., 51, 3754(2002).

68. E. H. Land, U.S.Patent 2123902 (1938).

69. S. Mamada and T. Kimura, Polym. Prepr. Jpn., 51, 726, 2951 (2002).

70. "Physical Properties of Crystals", Clarendon Press, Oxford, London (1993).

71. T. Kawai, R. Iijima, Y. Yamamoto, and T. Kimura, J. Phys. Chem. B, 105, 8077 (2001).

72. T. Kawai, R. Iijima, Y. Yamamoto, and T. Kimura, Polymer, 43, 7301 (2002).

73. T. S. Suzuki and Y. Sakka, Jpn. J. Appl. Phys., 41, L1272 (2002).

74. T. S. Suzuki and Y. Sakka, Chem. Lett., 2002, 1204.

75. T. Kimura, T. Yamane, and M. Yamato, "Proc. of The 6th Meeting of Symposium on New Magneto-Science'02", Tsukuba, November, 2002, p.24.

76. W. R. Krigbaum, "Polymer Liquid Crystals" A. Ciferri, W. R. Krigbaum, and R. B. Meyer Eds., Academic Press, London, Ch. 10 (1982).

77. G. Maret and A. Blumstein, Mol. Cryst. Liq. Cryst., 88, 295 (1982).

78. F. Hardouin, M. F. Achard, H. Gasparoux, L. Liebert, and L. Strzelecki, J. Polym. Sci., Polym. Phys. Ed., 20, 975 (1982).

79. J. S. Moore and S. I. Stupp, Macromolecules, 20, 282 (1987).

80. A. Anwer and A. H. Windle, Polymer, 32, 103 (1991).

81. S. Kossikhina, T. Kimura, E. Ito, and M. Kawahara, Polym. Eng. Sci., 37, 396 (1997).

82. S. Kossikhina, T. Kimura, E. Ito, and M. Kawahara, Polym. Eng. Sci., 38, 914 (1998).

83. T. Shimoda, T. Kimura, and E. Ito, Macromolecules, 30, 5045 (1997).

84. F. Oda, S. Nozawa, M. Hayashi, I. Shiga, M. Kimura, and K. Kajimura, Kobunshi Ronbunshu, 46, 101 (1989).

85. T. Kimura, T. Maeda, H. Sata, M. Yamato, and E. Ito, Polym. J., 27, 247 (1995). 
86. T. Kimura, H. Sata, and E. Ito, Polym. J., 30, 455 (1998).

87. T. Kimura, S. Kossikhina, T. Shimoda, E. Ito, and M. Kawahara, Mol. Cryst. Liq. Cryst., 318, 279 (1998).

88. T. Sun, S. K. Bhattacharya, R. W. Lenz, and R. S. Porter, J. Polym. Sci., Polym. Phys. Ed., 28, 1677 (1990).

89. M. Yamato and T. Kimura, Chem. Lett., 2000, 1296.

90. H. Sata, T. Kimura, S. Ogawa, M. Yamato, and E. Ito, Polymer, 37, 1879 (1996).

91. H. Sata, T. Kimura, S. Ogawa, and E. Ito, Polymer, 39, 6325 (1998).

92. H. Ezure, T. Kimura, S. Ogawa, and E. Ito, Macromolecules, 30, 3600 (1997).

93. T. Kawai and T. Kimura, Polymer, 41, 155 (2000).

94. T. Kimura, T. Kawai, and Y. Sakamoto, Polymer, 41, 809 (2000).

95. T. Kimura, M. Yamato, W. Koshimizu, and T. Kawai, Chem. Lett., 1999, 1057.

96. H. H. Shao, H. Gang, and E. B. Sirota, Phys. Rev. E., 57, R6265 (1998).

97. F. Kimura, T. Kimura, T. Kawata, M. Iwasaka, and S. Ueno, Trans. MRS-J., 27, 133 (2002).

98. M. Yamato and T. Kimura, Trans. MRS-J, 27, 117 (2002).

99. H. Aoki, M. Yamato, and T. Kimura, Chem. Lett., 2001, 1140.

100. T. Kimura, M. Yamato S. Endo, F. Kimura, H. Sata, H. Kawasaki, and Y. Shinagawa, J. Polym. Sci. B, 39, 1942 (2001).

101. T. Kawai, Y. Sakamoto, and T. Kimura, Mater. Trans. JIM, 41, 955 (2000).

102. W. Brostow, in "Physical Properties of Polymers Handbook", J. E. Mark Ed., American Institute of Physics Press, Woodbury, N.Y., 1996.

103. B. Wunderlich and J. Grebowicz, Adv. Polym. Sci., 60/61, 1 (1984).

104. T. Kawai and T. Kimura, "Proc. 3rd Meeting on New Magneto-Science'99”, 392 (1999).

105. F. Kimura, T. Kimura, M. Iwasaka, and S. Ueno, Polym. Prepr. Jpn., 51, 2070 (2002).

106. H. Sata, Thesis, Tokyo Metropolitan University, 1998.

107. T. Kimura, Jpn. J. Appl. Phys., 40, 6818 (2001).

108. H. Inaba, K. Tozaki, S. Wang, H. Hayashi, and T. Kimura, "Proc. 5th Meeting on New Magneto-Science'01", 283 (2001).

109. H. Inaba, K. Tozaki, S. Hosaka, H. Hayashi, and T. Kimura, "Proc. 5th Meeting on New Magneto-Science'01", 286 (2001).

110. Y. Sakamoto, T. Kawai, and T. Kimura, Trans. MRS-J., 25, 73 (2000).

111. R. P. de Daubeny, C. W. Bunn, and C. J. Brown, Proc. R. Soc., (London) A 226, 531 (1954).

112. T. Kimura and E. Ito, Mater. Res. Soc. Symp. Proc., 598,
BB3.1.1-3.1.5 (2000).

113. A. Miyake, J. Polym Sci., 38, 479 (1959).

114. M. Kobayashi, K. Akita, and H. Tadokoro, Makromol. Chem., 118, 324 (1968).

115. I. Ouchi, M. Hosoi, and S. Shimotsuma, J. Appl. Polym. Sci., 21, 3445 (1977).

116. P. C. Painter and J. L. Koenig, J. Polym. Sci., B, 15, 15, 1885 (1977).

117. S. -B. Lin and J. L. Koenig, J. Polym. Sci., B, 21, 2067 (1983).

118. K. Tashiro, K. Imanishi, Y. Izumi, M. Kobayashi, K. Kobayashi, M. Satoh, and R. S. Stein, Macromolecules, 28, 8477 (1995).

119. H. Zhang, A. Rankin, and I. M. Ward, Polymer, 37, 1079 (1996).

120. F. Kimura, T. Kimura, A. Sugisaki, M. Komatsu, H. Sata, and E. Ito, J. Polym. Sci., B, 35, 2741 (1997).

121. T. Kimura, H. Ezure, S. Tanaka, and E. Ito, J. Polym. Sci., B, 36, 1227 (1998).

122. H. Aoki, M. Yamato, and T. Kimura, Polym. Prepr. Jpn., 51, 444 (2002).

123. S. Kamata, S. Kadowaki, Y. Saito, T. Kohmoto, and Y. Yamamoto, Fiber Prepr. Jpn., 53, 99 (1997).

124. T. Kawai, T. Kimura, and Y. Yamamoto, Trans. MRS-J., 27, 125 (2002).

125. M. Aramaki, T. Kawai, and T. Kimura, Polym. Prepr. Jpn., 51, 452 (2002).

126. T. Kawai and T. Kimura, Macromolecules, 33, 8421 (2000).

127. J. K. Kruger, L. Peetz, W. Wildner, and M. Pietralla, Polymer, 21, 620 (1980).

128. J. K. Kruger, Solid State Commun., 30, 43 (1979).

129. M. Nakatsuka, K. Fujioka, T. Kanabe, and H. Fujita, J. Cryst. Growth, 171, 531 (1997).

130. C. Rosenblatt, Phys. Rev. A: At., Mol., Opt. Phys., 24, 2236 (1981).

131. K. S. Krishnan, B. C. Guha, and S. Banerjee, Philos. Trans. R. Soc. (London) A 231, 235 (1933).

132. T. Kimura, Mat. Trans. JIM, accepted.

133. Y. Ikezoe, N. Hirota, J. Nakagawa, and K. Kitazawa, Nature, 393, 749 (1998).

134. Y. Ikezoe, T. Kaihatsu, T. Suetsuna, H. Uetake, N. Hirota, and K. Kitazawa, "Proc. 3rd Meeting on New MagnetoScience'99", 400 (1999).

135. N. Hirota, Y. Ikezoe, H. Uetake, T. Kaihatsu, T. Takayama, T. Mori, H. Nakamura, and K. Kitazawa, "Proc. 5th Meeting on New Magneto-Science'01”, 160 (2001).

136. T. Kimura, S. Mamada, and M. Yamato, Chem. Lett., 2000, 1294.

137. M. Yamato, H. Nakazawa, and T. Kimura, Langmuir, 18, 9609 (2002).

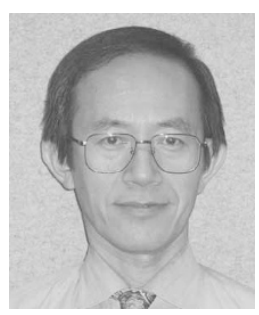

Tsunehisa Kimura was born in Kanagawa in 1952. He received his Ph.D. from Kyoto University in 1981 for theoretical study on dilute polymer solutions of block copolymers and ring polymers. He joined Asahi Chemical in 1982 and was involved in various research fields including design of film fabrication process, NMR, etc. Then, he quit Asahi Chemical in 1990 and joined McGill University as a post-doctoral fellow, where he worked on high-yield pulp and liquid crystals. From 1994 to 2002, he was Associate Professor at Tokyo Metropolitan University, and currently Professor at the same university. Since 1999 he has been a project leader of the Research for the Future Program by Japan Society for the Promotion of Science. He received The Award of the Society of Polymer Science, Japan (2001). His current research interests include magnetic effects on feeble magnetic materials and its application to polymer processing. 